\title{
A multi-resolution method for climate system modeling: Application of Spherical Centroidal Voronoi Tessellations
}

\author{
Todd Ringler · Lili Ju · Max Gunzburger
}

Received: date / Accepted: date

\begin{abstract}
During the next decade and beyond, climate system models will be challenged to resolve scales and processes that are far beyond their current scope. Each climate system component has its prototypical example of an unresolved process that may strongly influence the global climate system, ranging from eddy activity within ocean models, to ice streams within ice sheet models, to surface hydrological processes within land system models, to cloud processes within atmosphere models. These new demands will almost certainly result in the develop of multi-resolution schemes that are able, at least regional, to faithfully simulate these fine-scale processes. Spherical Centroidal Voronoi Tessellations (SCVTs) offer one potential path toward the development of robust, multi-resolution climate system component models. SCVTs allow for the generation of high quality Voronoi diagrams and Delaunay triangulations through the use of an intuitive, user-defined density function. In each of the examples provided, this method results in high-quality meshes where the quality measures are guaranteed to improve as the number of nodes is increased. Real-world examples are developed for the Greenland ice sheet and the North Atlantic ocean. Idealized examples are developed for ocean-ice shelf interaction and for regional atmospheric modeling. In addition to defining, developing and exhibiting SCVTs, we pair this mesh generation
\end{abstract}

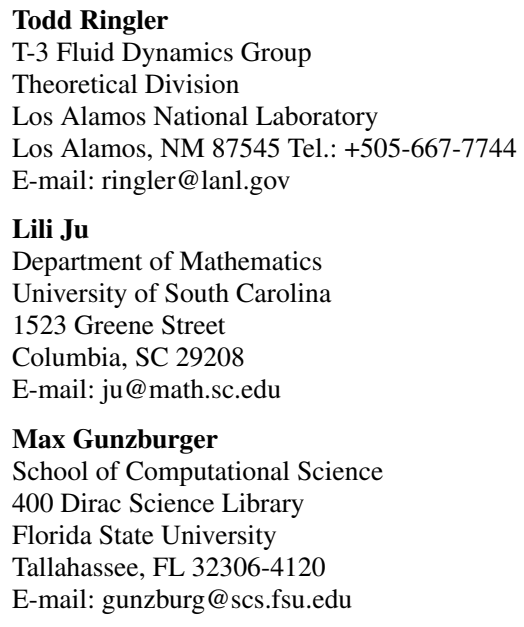


technique with a previously developed finite-volume method. Our numerical example is based on the nonlinear shallow-water equations spanning the entire surface of the sphere. This example is used to elucidate both the potential benefits of this multi-resolution method and the challenges ahead.

Keywords Voronoi $\cdot$ Delaunay $\cdot$ climate modeling $\cdot$ multi-resolution

\section{Introduction}

Climate system models (CSMs) are an increasingly important tool for assessing anthropogenic climate change. CSMs, along with observations and theory, form the basis for the IPCC Working Group 1 Assessment Reports that detail the anticipated consequences of rising concentrations of atmospheric greenhouse gases [1]. While CSMs have been highly successful in interpreting observations, confirming theory and providing gross estimates of climate sensitivity, the climate modeling community will be challenged in the coming decade to extend the utility of CSMs well beyond their current scope.

At least two drivers are pushing CSMs into new and expanding roles. The first is the increasingly urgent need to resolve scales and processes that are far beyond the current scope of these models. There are likely to be unresolved processes and currently misrepresented processes that have significant influence on the global climate system. Every component of the Earth system has its own prototypical example, ranging from eddy activity within ocean models [13], to ice streams within ice sheet models [15], to surface hydrological processes within land system models [22], to cloud processes with atmosphere models [32]. Each of these processes are not faithfully included in IPCC-class CSMs primarily due to lack of resolution; the degrees of freedom required to comprehensively simulate these processes are computationally prohibitive given the current (and foreseeable) resources.

The second driver pushing the evolution CSMs is the rapidly growing demand for highfidelity assessments of regional climate change driven by increasing concentrations of atmospheric greenhouse gases. As appreciation for the possible consequences of anthropogenic climate change improves, we are confronted with the need to characterize the regional aspects of climate change in order to support mitigation strategies. As indicated by the last chapter of the IPCC WG1 Fourth Assessment Report (AR4), the push in this direction is already underway [1]. To be successful in providing the relevant information regarding regional climate impacts, CSMs will require significant increases in resolution, at least regionally, along with the incorporation of new processes.

Climate system model components are presently utilizing various types of quasi-uniform tessellations (aka grids or meshes) to discretize the surface of the sphere. These quasiuniform tessellations are a significant improvement over their predecessor, latitude-longitude grids, by removing both the strong grid-pole singularities and the accompanying numerical filters required to regularize these singularities. Various types of meshes have been proposed as alternatives to the traditional latitude-longitude grid. For example, the cubedsphere, which offers the same topological structure as the latitude-longitude grid without the strong pole singularities, has been successfully implemented in various efforts [20]. Voronoi tessellations (aka geodesic, icosahedral or hexagonal grids) have sometimes been chosen for their remarkable uniformity and isotropy [27]. And finally, closely related to these Voronoi tessellations are the Delaunay triangulations that have been successfully implemented in an idealized setting and are now being integrated into full CSMs [4]. While each of these methods have successfully removed the grid pole singularities associated with latitude-longitude 
grids, it is not clear that any of these methods, as presently formulated, will be able to meet the challenges outlined above.

By their nature, quasi-uniform tessellations imply substantial increase in computational costs to increase horizontal resolution. A halving of the nominal grid spacing implies an increase in computational cost of approximately a factor of eight; a factor of four arises from doubling the degrees of freedom in each of the horizontal directions and a factor of two arises due to halving the time step. The computational burden associated with increasing resolution everywhere within the domain quickly exhausts available computational resources. For example, producing eddy-resolving ocean simulations as a part of century-long coupled climate simulations are impracticable now and will likely continue to be so for at least the next decade or more. The current NCAR Coupled Climate System Model [5] uses an ocean component model with a $320 \times 384$ grid and a time step of approximately one hour. The eddy-resolving version of this ocean model uses a $3600 \times 2400$ grid and a time step of approximately six minutes [21]. The two configurations differ by a factor of about 1000 in terms of their computational burden. Similarly daunting computational burdens are found in ice sheet modeling, surface hydrology modeling and atmospheric modeling.

The obvious implication here is that CSMs will not be able to fulfill their expanding roles by solely using quasi-uniform tessellations. The corollary to this assertion is that multiresolution schemes will be required if CSMs are to meet the growing challenges over the next decade. A host of scientific complexities arise as we begin to contemplate the construction of a multi-resolution IPCC-class climate system model.

The recent work of St-Cyr et al. [30] clearly indicates that a successful multi-resolution scheme requires attention to the combination of method and mesh. The authors develop a multi-resolution mesh by implementing an adaptive, hierarchical nesting technique in which quadrilateral elements are bisected to locally increase resolution. When this adaptive meshing technique was used in combination with a high-order spectral method, the resulting multi-resolution scheme produced positive results. Alternatively, when the same technique was used in combination with a low-order finite-volume technique, the results were equivocal at best; adding degrees of freedoms did not reduce numerical solution error. The implication is that robust, multi-resolution climate system components will require close attention to both the quality of the variable-resolution meshes and to the numerical techniques we place "on top" of these meshes.

An alternative to hierarchical nesting is to produce a smoothly-varying tessellation. By their design, smoothly varying tessellations provide strong control over the spatial patterns of truncation error. While this control may be superfluous when used in combination with high-order methods, it may prove to be critically important when used with the low-order, finite-volume methods that are ubiquitous in IPCC-class component models [1]. The primary purpose of this paper is to develop a class of robust, variable-resolution meshes, called Spherical Centroidal Voronoi Tessellations, that have the requisite characteristics necessary to meet the present and future challenges of climate system modeling.

Spherical Centroidal Voronoi Tessellations (SCVTs) contain a host of qualities that should produce tangible benefits in the context of climate modeling. First, SCVTs are a superset of the quasi-uniform Voronoi tessellations currently being used in the climate modeling community. Thus, SCVTs are a logical extension to meshes already being utilized. Second, as discussed in Section 2, even non-uniform SCVTs always produce smoother, more locally-uniform meshes as the degrees of freedom are increased. The implication here is clear; SCVTs offer a robust means of producing multi-resolution meshes that are guaranteed to increase in quality as computational resources grow. As discussed in Section 3, the technique to produce variable-resolution SCVTs is intuitive and straightforward to im- 
plement. And finally, each SCVT is uniquely associated with a Delaunay triangulation. The positive attributes associated with the SCVTs are also present in the associated Delaunay triangulation. While a tremendous amount of work is required to translated these positive attributes into robust climate simulations, we begin the task here by taking two steps. First, we develop example meshes for several types of climate system components to demonstrate the methods ability to produce high-quality, variable-resolution meshes in a diversity of systems. Second, we will demonstrate the ability of these meshes to reduce solution errors, at least locally, in the context of the shallow-water system.

The two primary purposes of this paper are the following: 1) to introduce the climate modeling community to the basic principles of SCVT and 2) to highlight the broad applicability of SCVT to climate system modeling. Section 2 introduces and develops the mathematical foundation for SCVTs. Section 3 develops real-world SCVTs in the context of ice sheet and ocean modeling. Section 4 combines our SCVT technique for generation of multi-resolution meshes with a low-order finite-volume technique to produce a prototype multi-resolution scheme that is broadly applicable to climate system modeling. We look toward future developments of SCVT and draw some conclusions in Section 5.

\section{Centroidal Voronoi Tessellations}

\subsection{Definitions}

Let $\Omega$ denote an open domain or a piecewise smooth hyper-surface in $\mathbb{R}^{d}$ and $\|\cdot\|$ the corresponding standard Euclidean metric for $\mathbb{R}^{d}$. Given a set of distinct points $\left\{\mathbf{x}_{i}\right\}_{i=1}^{n} \subset \Omega$, we define

$$
V_{i}=\left\{\mathbf{y} \in \Omega \mid\left\|\mathbf{x}_{i}-\mathbf{y}\right\|<\left\|\mathbf{x}_{j}-\mathbf{y}\right\| \quad \text { for } \quad j=1, \ldots, n \quad \text { and } j \neq i\right\}
$$

for $i=1, \ldots, n$. Clearly, $\left\{V_{i}\right\}_{i=1}^{n}$ forms a tessellation of $\Omega$, i.e., the union of $\bar{V}_{i}$ spans $\bar{\Omega}$ written as $\cup_{i=1}^{n} \bar{V}_{i}=\bar{\Omega}$. We refer to $\left\{V_{i}\right\}_{i=1}^{n}$ as the Voronoi tessellation or Voronoi diagram [23] of $\Omega$ associated with the point set $\left\{\mathbf{x}_{i}\right\}_{i=1}^{n}$. A point $\mathbf{x}_{i}$ is called a generator and a subregion $V_{i}$ is referred to as the Voronoi region corresponding to the generator $\mathbf{x}_{i}$. The duality (in a graph-theoretical sense) of a Voronoi tessellation of $\Omega$ is the well-known Delaunay tessellation which often consists of triangles/tetrahedra. Algorithms for the construction of corresponding Voronoi diagrams and Delaunay triangulations have been well developed [23, 24].

Given a density function $\rho(\mathbf{x})$ defined on $\Omega$, for any region $V \subset \Omega$, we call $\mathbf{x}^{c}$ the constrained mass centroid of $V$ with respect to $\Omega$ if

$$
\mathbf{x}^{c}=\arg \min _{\mathbf{x} \in V} \int_{V} \rho(\mathbf{y})\|\mathbf{y}-\mathbf{x}\|^{2} \mathrm{~d} \mathbf{y} .
$$

The existence of solutions of (1) can be easily obtained using the continuity and compactness of the object function; however, solutions may not be unique. It is worth noting that if $\Omega$ is an open domain or a flat hyper-surface, then $\mathbf{x}^{c}$ coincides with $\mathbf{x}^{*}$, the standard mass centroid of $V$ defined by

$$
\mathbf{x}^{*}=\frac{\int_{V} \mathbf{y} \rho(\mathbf{y}) \mathrm{d} \mathbf{y}}{\int_{V} \rho(\mathbf{y}) \mathrm{d} \mathbf{y}}
$$


that is much easier to compute.

A Voronoi tessellation of $\Omega$ is called a constrained centroidal Voronoi tessellation (CCVT) [8] if and only if the points $\left\{\mathbf{x}_{i}\right\}_{i=1}^{n}$ which serve as the generators of the associated Voronoi tessellation $\left\{V_{i}\right\}_{i=1}^{n}$ are also the constrained mass centroids $\left\{\mathbf{x}^{c}\right\}_{i=1}^{n}$ of those regions, i.e., if and only if

$$
\mathbf{x}_{i}=\mathbf{x}_{i}^{c}, \quad i=1, \ldots, n .
$$

We often refer to the relation (2) as the CVT property. The dual Delaunay grid is then called constrained centroidal Voronoi Delaunay triangulation (CCVDT). We remark that when $\Omega$ is a open domain in $\mathbb{R}^{d},\left\{\mathbf{x}_{i}, V_{i}\right\}_{i=1}^{n}$ is often just called a centroidal Voronoi tessellation (CVT) [7].

A very important case should be specially addressed for the application of CVT/CCVT to climate system modeling, that is, $\Omega$ denotes the surface of a sphere in $\mathbb{R}^{3}$ or part of it. In this case, we often refer to $\left\{\mathbf{x}_{i}, V_{i}\right\}_{i=1}^{n}$ as a spherical centroidal Voronoi tessellation (SCVT). It is easy to verify that

$$
\mathbf{x}^{c}=r \frac{\mathbf{x}^{*}}{\left\|\mathbf{x}^{*}\right\|}
$$

where $r$ denotes the radius of the sphere, so that $\mathbf{x}^{c}$ can be easily computed by first determining $\mathbf{x}^{*}$.

General Voronoi tessellations do not satisfy the CVT property, see Figure 1 for a illustration. A square domain is randomly seeded with ten points (dots in Figure 1, left panel). These ten points serve as generators for the Vonoroi tessellation (cell boundaries in Figure 1, left panel). For each Voronoi region, the standard mass centroid (open circles in Figure 1, left panel) is computed. As discussed below in Section 2.3, a simple iterative calculation regularizes the initial Voronoi diagram on the left to the diagram shown to the right. In this example we used a constant density to compute the standard mass centroid; a variable density field would have biased the resulting generator points toward the region of high density. This relationship between density and generator position is the key aspect of this grid generation method.

\subsection{Properties}

Given any set of points $\left\{\widetilde{\mathbf{x}}_{i}\right\}_{i=1}^{n}$ on $\Omega$ and any tessellation $\left\{\widetilde{V}_{i}\right\}_{i=1}^{n}$ of $\Omega$, we define the energy functional

$$
\mathscr{K}\left(\left\{\widetilde{\mathbf{x}}_{i}, \widetilde{V}_{i}\right\}_{i=1}^{n}\right)=\sum_{i=1}^{n} \int_{\widetilde{V}_{i}} \rho(\mathbf{y})\left\|\mathbf{y}-\widetilde{\mathbf{x}}_{i}\right\|^{2} d \mathbf{y}
$$

The energy is often referred to as some physical quantity such as variance, cost, distortion error, or mean square error in practical applications. A priori, there is no assumed relation between the point set $\left\{\widetilde{\mathbf{x}}_{i}\right\}_{i=1}^{n}$ and the tessellation $\left\{\widetilde{V}_{i}\right\}_{i=1}^{n}$. However, it can be shown that $\mathscr{K}(\cdot)$ is minimized only if $\left\{\widetilde{\mathbf{x}}_{i}, \widetilde{V}_{i}\right\}_{i=1}^{n}$ is a CVT/CCVT [7,8]. Thus, CVTs/CCVTs are Voronoi tessellations for which the generators are, in some sense, optimally distributed.

Let us set $\tilde{d}=d$ if $\Omega$ is an open domain and $\tilde{d}=d-1$ if $\Omega$ is a hyper-surface in $\mathbb{R}^{d}$. Specially, for SCVTs, we have $\tilde{d}=2$. As a consequence, CVT/CCVT meshes in $\mathbb{R}^{d}$ have many good geometric properties, including the following $[7,8,10]$ :

- For a constant density function, the generators $\left\{\mathbf{x}_{i}\right\}_{i=1}^{m}$ are uniformly distributed across $\Omega$. 
- Most of Voronoi regions are (nearly) congruent [12,7]. Specially, for SCVTs, they are primarily convex spherical hexagons.

- The mesh size $h$ is approximately proportional to $n^{-1 / \tilde{d}}$

- For a non-constant density function, the generators $\left\{\mathbf{x}_{i}\right\}_{i=1}^{m}$ are still locally uniformly distributed and it is conjectured (and computationally verified) that, asymptotically,

$$
\frac{h_{V_{i}}}{h_{V_{j}}} \approx\left(\frac{\rho\left(\mathbf{x}_{j}\right)}{\rho\left(\mathbf{x}_{i}\right)}\right)^{\frac{1}{d+2}} .
$$

- The relationship between the relative sizes of Voronoi regions (i.e. grid cells) is controlled entirely by the specified density function.

- CVT/CCVT generators tend to accumulate in regions having relatively high values of $\rho$ while remaining locally very regular.

- Thus, in principle, one could control the distribution of generators to minimize the error (either locally or globally) in the solution of a partial differential equation by, e.g., connecting the density function $\rho(\mathbf{x})$ to some a priori or a posteriori error estimates.

\subsection{Algorithms}

Construction of CVT/CCVT is usually done by either probabilistic methods typified by MacQueen's random algorithm [19] (which is a simple iteration between sampling and averaging points) or deterministic methods typified by Lloyd iteration [18] (which is a simple iteration between constructing Voronoi diagrams and mass centroids). Due to the low convergence rate of MacQueen's method [19], much attention has been focused on Lloyd method described below:

Algorithm 1 (Lloyd Method) Given a domain $\Omega$, a density function $\rho(\mathbf{x})$ defined on $\Omega$, and a positive integer $n$.

0. Select an initial set of $n$ points $\left\{\mathbf{x}_{i}\right\}_{i=1}^{n}$ on $\Omega$;

1. Construct the Voronoi regions $\left\{V_{i}\right\}_{i=1}^{n}$ of $\Omega$ associated with $\left\{\mathbf{x}_{i}\right\}_{i=1}^{n}$;

2. Determine the (constrained) mass centroids of the Voronoi regions $\left\{V_{i}\right\}_{i=1}^{n}$; these centroids form the new set of points $\left\{\mathbf{x}_{i}\right\}_{i=1}^{n}$;

3. If the new points meet some convergence criterion, return $\left\{\left(\mathbf{x}_{i}, V_{i}\right)\right\}_{i=1}^{n}$ and terminate; otherwise, goto step 1.

Referring to Figure 1, the process is as follows: The initial point set (shown as dots in left panel) represents Step 0. Step 1 is shown by the solid cell boundary lines in Figure 1 (left panel). Step 2, the location of the cell centroids, is shown by the open circles in Figure 1 (left panel) and form the new point set from which we compute the new Voronoi diagram. The final result, after satisfying the convergence criterion in Step 3 is shown in Figure 1 (right panel).

\subsection{Quality Measure of Voronoi Cells and Delaunay Triangles}

For the Voronoi cell $V_{i}$ associated with the generator $\mathbf{x}_{i}$, we define its size to be

$$
h_{V_{i}}=2 \max _{\mathbf{y} \in V_{i}}\left\|\mathbf{x}_{i}-\mathbf{y}\right\| \text {. }
$$


Then $h_{\max } / h_{\min }$ can be used to measure the global non-uniformality of the given Voronoi mesh where $h_{\text {max }}=\max _{i} h_{V_{i}}$ and $h_{\text {min }}=\min _{i} h_{V_{i}}$. In order to measure the local unformality or quality of Voronoi cells of the SCVT, we use the following $\sigma$ measure [9]. For the Voronoi cell $V_{i}$ associated with the generator $\mathbf{x}_{i}$,

$$
\sigma\left(V_{i}\right)=\frac{\min _{j}\left\|\mathbf{x}_{i}-\mathbf{x}_{j}\right\|}{\max _{j}\left\|\mathbf{x}_{i}-\mathbf{x}_{j}\right\|}
$$

where $\mathbf{x}_{j}$ 's denote Voronoi neighbors of $\mathbf{x}_{i}$. Clearly $0<\sigma \leq 1$ and $\sigma=1$ corresponds to the equilateral polygons. We then set

$$
\sigma_{\min }=\min _{i} \sigma\left(V_{i}\right) \quad \text { and } \quad \sigma_{\text {avg }}=\frac{1}{n} \sum_{i} \sigma\left(V_{i}\right)
$$

where $n$ denotes the number of Voronoi cells. $\sigma_{\min }$ measures the quality of the worst Voronoi cell and $\sigma_{a v g}$ measures the average quality of the Voronoi mesh.

The size of a triangle $T, h_{T}$ is defined to be its longest side length. We apply the commonly used $q$-measure [11] to evaluate the quality of the dual triangular mesh (Delaunay triangles), where, for any triangle $T, q$ is defined to be twice the ratio of the radius $R_{T}$ of the largest inscribed circle and the radius $r_{T}$ of the smallest circumscribed circle, i.e.,

$$
q(T)=2 \frac{R_{T}}{r_{T}}=\frac{(b+c-a)(c+a-b)(a+b-c)}{a b c},
$$

where $a, b$, and $c$ are side lengths of $T$. Clearly $0<q \leq 1$ and $q=1$ corresponds to the equilateral triangle. For a given triangulation, $\mathscr{T}$, composed of $m$ triangles, we define

$$
q_{\text {min }}=\min _{T \in \mathscr{T}} q(T) \quad \text { and } \quad q_{\text {avg }}=\frac{1}{m} \sum_{T \in \mathscr{T}} q(T) .
$$

$q_{\text {min }}$ measures the quality of the worst triangle and $q_{\text {avg }}$ measures the average quality of the triangular mesh $\mathscr{T}$.

It is worth noting that the energy $\mathscr{K}$ associated with the Voronoi tessellation $\left\{\left(\mathbf{x}_{i}, V_{i}\right)\right\}_{i=1}^{n}$ decreases monotonically during the Lloyd iteration if $\left\{\left(x_{i}, V_{i}\right)\right\}_{i=1}^{n}$ has not reached a CVT/CCVT yet. In certain systems, or with certain methods, we may require nodes to be located on the boundary of the problem domain $\Omega$. CVTs/CCVTs and the above construction algorithm can be easily generalized so that some of the generators are constrained to lie on the boundary $\partial \Omega[10,16]$.

\section{Example Meshes}

\subsection{Land Ice: Greenland}

\subsubsection{Motivation}

The Greenland and Antarctic Ice Sheets are characterized by a wide range of spatial and temporal scales. In terms of spatial scales, each of these ice sheets span several thousand kilometers. Interior regions of these ice sheets are characterized by relatively broad spatial scales on the order of $100 \mathrm{~km}$ or more. These interior regions are generally areas of net accumulation of mass due to atmospheric precipitation of water. As this net source of water is exported toward the ocean in the form of ice, relatively fast-moving ice streams form 
within each catchment zone. Not unlike their liquid water counterparts on land, these ice streams are long and thin with along-stream scales of several hundred kilometers and crossstream scales often less than $10 \mathrm{~km}$ ([15]). In addition, the shear zone separating the fastmoving ice streams from the adjacent nearly-stationary ice is characterized by scales of 1 $\mathrm{km}$ or less. These ice streams transport the majority of ice volume from Greenland and Antarctica into the surrounding ocean [25]. As a result, robust predictions of sea-level rise will require an accurate simulation of ice stream dynamics. In fact, the recent IPCC WG1 AR4 document declined to draw substantive conclusions on the likelihood of rapid sea level rise during the 21 st century because, in part, ice stream dynamics are not include in current ice sheet models [1].

In addition to the kinematically-driven need for locally enhanced resolution, there is also a desire for increased resolution at the ice margin due to significant seasonal ablation. This is particularly relevant for Greenland where intense melting occurs annually below 1200 $\mathrm{m}$. The subsequent transport of this meltwater to the bottom of the ice sheet may have a strong impact on basal sliding processes [3]. In the context of ice sheet modeling, we see both kinematics and physics as driving the need for locally-enhanced resolution. Spatial resolutions below $1 \mathrm{~km}$ might be required for the accurate representation of these processes. When considering an entire ice sheet, a uniform mesh of $1 \mathrm{~km}$ combined with the emerging three-dimensional Stokes solvers is not computationally tractable. So, instead, we turn to variable-resolution SCVTs to discretize this system.

\subsubsection{Proxy for SCVT Density}

In this example we will generate a mesh of Greenland that places enhanced resolution at the ice margin. Extensions of this technique to accommodate ice streams are straightforward and we will explore such an extension in Section 3.3. Our target resolution for this grid is 2 $\mathrm{km}$ in the vicinity of the ice margin and $50 \mathrm{~km}$ in the interior. Our estimates suggest that the resulting mesh will be computationally tractable for climate change simulations, even when used in combination with a full three-dimensional Stokes solver. Figure 2 (left panel) shows an observational estimate of Greenland ice thickness at a spatial resolution of $5 \mathrm{~km} \mathrm{[2].} \mathrm{We}$ use the distribution of ice thickness in two ways. First, this data set allows us to define the location of the ice boundary of Greenland as a set of piece-wise linear loops (see Figure 2 (right panel)) within which we will develop the SCVT. Second, within each loop we define the SCVT density function as

$$
\rho=\min \left(25^{\frac{1}{4}},\left(\frac{1}{H}\right)^{0.1}\right)
$$

We limit the upper bound of SCVT density to maintain the ratio of nominal maximum grid spacing to nominal minimum grid spacing to be approximately 25 . Figure 3 depicts the resulting Voronoi diagram using 29747 nodes, resulting in a minimum resolution of approximately $4 \mathrm{~km}$. In Figure 3 we find broad regions of low resolution throughout the interior of the ice sheet with the vast majority of the nodes position near the ice margin. 9 out 10 cells are spaced less then $10 \mathrm{~km}$ from their neighbors. We progressively add nodes into the domain until our target minimum grid resolution of $2 \mathrm{~km}$ is reached. Figure 4 (top panel) compares a section of the low resolution mesh to its high resolution counterpart Figure 4 (bottom panel) that is composed of 112896 nodes. As expected, the majority of the nodes are positioned near the ice margin. Table 1 presents the global quality metrics for the Greenland SCVTs. The quality histograms of the SCVTs are shown in Figure 5. The bulk measures of 
uniformity shown in Table 1 show improvement in every category as resolution is increased. The histograms shown in Figure 5 indicate a systematic shift toward higher mesh quality with increasing degrees of freedom. Equally important, as the number of nodes is increased the histograms exhibit a noticeable reduction in the proportion of cells residing in the "lowquality" end of the histograms.

\begin{tabular}{|l|c|c|r|r|c|c|}
\hline \# of Generators & $\sigma_{\min }$ & $\sigma_{\text {avg }}$ & $h_{\max } / h_{\min }$ & \# of Triangles & $q_{\min }$ & $q_{\text {avg }}$ \\
\hline \hline 29474 & 0.131 & 0.695 & 66.49 & 53949 & 0.345 & 0.928 \\
\hline 112896 & 0.148 & 0.741 & 73.23 & 215650 & 0.411 & 0.945 \\
\hline
\end{tabular}

Table 1: Mesh information of SCVTs for the Greenland.

\subsection{Ocean: North Atlantic}

\subsubsection{Motivation}

Incorporating eddies into IPCC-class global ocean simulations remains a computational challenge. Eddy-resolving simulations typically require grid resolutions of approximately $10 \mathrm{~km}$, implying approximately $5 \mathrm{e} 6$ degrees of freedom to span the global ocean surface. This is in stark contrast to typical IPCC simulations that currently use approximately 5e 4 degrees of freedom to cover the same extent. The factor of 100 separating the two simulations is compounded by another factor of 10 since eddy-resolving simulations require a significantly shorter time step. The 1000-fold increase in computational burden to move from resolutions presently used in IPCC-class simulations to global, eddy-resolving resolutions is currently beyond reach and will likely remain so for a decade or more.

An alternative and computationally-tractable approach is to employ variable-resolution grids, such as SCVTs, to permit eddy-resolving resolutions at targeted locations. These variable-resolution grids could be employed in limited area domains or as part of a global ocean simulation. The ability to readily generate variable-resolution meshes for the global ocean system allows us to consider the notion of an optimal spatial allocation of computation resources. In addition, the scientific study of certain processes would certainly benefit from the ability to support eddy activity in certain regions while maintaining a global ocean domain. Two supporting examples include the role of eddies in the North Atlantic on the thermohaline circulation and the role of eddies in the Southern Ocean in mediating the ocean's response to changes in wind stress forcing.

\subsubsection{Proxy for SCVT density}

In this example we will derive a variable-resolution mesh of the North Atlantic Ocean with sufficient resolution to resolve eddies within the major current systems. The domain is identical to the nominal 1/10 degree eddy-resolving simulation discussed in Smith et al. [28]. Using data from these simulations, we compute the time-mean kinetic energy of the surface currents, as shown in Figure 6. As can be seen in Figure 6, the regions of eddy activity are extremely localized. Based on the kinetic energy, $K E$, we defined the density function as

$$
\rho=\max \left[0.2, \frac{K E}{K E_{\max }}\right]^{8}
$$


where $K E_{\max }$ is the maximum kinetic energy in the domain. The lower bound of 0.2 insures that a minimum resolution is maintained in the quiescent regions. This choice leads to a ratio of minimum to maximum grid spacing of approximately 10 . In addition, we enhance the density function near the land-sea interface to insure that the boundary is adequately resolved. While we want the mesh to capture these regions of high activity via enhanced resolution, we also recognize the need to expand this region to allow eddies to travel uninhibited by grid resolution. As such, we applied a substantial amount of Laplacian smoothing to our density function (approximately 20 passes) to expand and smooth the regions of enhanced resolution. [Note that the RMS of sea-surface height is also an accurate reflection of mesoscale ocean variability and we have developed global ocean SCVT based on TOPEX remote sensing of sea-surface height.]

As with the Greenland example, we produced a continuous, piece-wise linear representation of the land-ocean boundary based on the land-sea mask used in the 0.1 degree simulation. This approach also identifies all islands. Islands with a circumference less than $10 \mathrm{~km}$ were discarded; the resulting domain contained 58 islands.

The Voronoi diagram shown in Figure 7 uses 40162 nodes. This results in a minimum grid resolution of approximately $20 \mathrm{~km}$. We continue to add nodes into the domain until we reach a minimum resolution of $10 \mathrm{~km}$. Closeups of this high resolution mesh using 157366 nodes and its low resolution counterpart are shown in Figure 8.

Table 2 presents the results of our North Atlantic SCVTs. The corresponding quality histograms are shown in Figure 9. As with the Greenland example, the quality measures show a systematic improvement as we increase the degrees of freedom.

\begin{tabular}{|l|c|c|r|r|c|c|}
\hline \# of Generators & $\sigma_{\min }$ & $\sigma_{\text {avg }}$ & $h_{\max } / h_{\min }$ & \# of Triangles & $q_{\min }$ & $q_{\text {avg }}$ \\
\hline \hline 40162 & 0.109 & 0.725 & 13.13 & 77017 & 0.148 & 0.937 \\
\hline 157366 & 0.186 & 0.763 & 10.53 & 308732 & 0.224 & 0.949 \\
\hline
\end{tabular}

Table 2: Mesh information of SCVTs for the North Atlantic.

\subsection{Ocean - Ice Shelf Interaction}

\subsubsection{Motivation}

Our final example couples ocean and ice domains in the context of ocean-ice shelf interaction. Ice shelves are ice flows that become ungrounded, buoyant and rest on top of ocean water. The location at which ice transitions from resting on bedrock to resting on ocean water is referred to as the grounding line. As the ice shelves are pushed outward into the ocean the ice is either melted along the ice-ocean interface or calves from the main shelf into icebergs. These ice shelves provide a significant buttressing force that resists the flow of upstream, grounded ice. As evidenced by the Larsen B ice shelf collapse, when the iceshelf buttressing force is removed the upstream ice flow can increase by several hundred percent [26]. The accurate simulation of ocean-ice shelf interaction is necessary in order to quantify the risk of rapid sea level rise [1].

The West Antarctic Ice Sheet (WAIS) is particularly relevant to the study of ocean-ice shelf interaction. Not only are ice shelves the primary outlet of grounded ice, the grounded ice frequently rests on bedrock that is increasingly below sea-level as one moves toward the 
ice interior region [29]. The physical geometry is such that an rapid erosion of the WAIS due to ocean-ice shelf interaction is a plausible scenario for the 21 st century.

Ice shelves connected to WAIS have spatial extents of more than $1000 \mathrm{~km}$ (e.g. the Ross Ice Shelf) down to less than $50 \mathrm{~km}$ (e.g. the Thwaites Ice Shelf). The embayments where this ice flows into the ocean have similar ranges in spatial scale. While the ice shelves are not as dynamically active as the ice streams that feed them, the structure and shape of the ice-ocean interface is a primary factor that drives mixing at this interface [14]. Grid resolutions of less than $5 \mathrm{~km}$ are often used when simulating ocean-ice shelf coupled dynamics. Furthermore, analysis of the global 1/10 degree ocean simulations in the vicinity of WAIS indicate that the transport of heat into these embayments may be eddy-driven and episodic (Maltrud, personal communication, 2007). Thus, resolving ocean eddies in and around these embayments will likely be required for robust simulations.

The horizontal discretization of this system is difficult because part of the domain will be ice (ice domain), part will be ocean (ocean domain) and part will be both ocean and ice (shelf domain). Furthermore, the characterization of a region as ice, ocean or shelf will evolve over the time scales of centuries to millennia. Due to this complexity and the fact that only limited work has been completed on modeling the coupled ocean-ice shelf system, we will explore techniques to discretize this system in an idealized setting. Figure 10 shows our idealized domain with a spatial extent of $1100 \mathrm{~km}$ by $550 \mathrm{~km}$. The domain is characterized by a region of grounded ice (to the left), a region of ocean (to the right) and an ice-shelf region (center). The ice domain includes an ice stream that feeds the shelf region. As indicated in the figure, a robust simulation of this system will require enhanced resolution in the vicinity of the ice shelf, ice stream and region of the ocean in proximity to the ice.

\subsubsection{Proxy for SCVT density}

In this idealize example our intent is to produce a tessellation with a minimum grid spacing of $2 \mathrm{~km}$ and a maximum grid spacing of $20 \mathrm{~km}$. As opposed to our other examples, we are not building our density function from a physical characteristic of the system. The generated density function has local maxima in the vicinity of the ice stream, in the region of the ice shelf and along the entire ocean-ice boundary. The resulting Voronoi diagram of this system using 9359 nodes is shown in Figure 11 with a close-up of the shelf region at both high and low resolution shown in Figure 12.

Table 3 presents the results on our SCVTs for the idealized ocean-ice sheet and corresponding quality histograms are shown in Figure 13. Yet again, the quality measure show increasing mesh quality with increasing degrees of freedom.

\begin{tabular}{|l|c|c|r|r|c|c|}
\hline \# of Generators & $\sigma_{\min }$ & $\sigma_{\text {avg }}$ & $h_{\max } / h_{\min }$ & \# of Triangles & $q_{\min }$ & $q_{\text {avg }}$ \\
\hline \hline 9359 & 0.275 & 0.735 & 8.91 & 18440 & 0.568 & 0.942 \\
\hline 37157 & 0.313 & 0.769 & 10.15 & 73765 & 0.626 & 0.951 \\
\hline
\end{tabular}

Table 3: Mesh information of SCVTs for ocean-ice shelf system.

\section{Example Numerical Method}

The large majority of numerical methods utilized in IPCC-class climate models were developed in the context of uniform meshes. Successfully implementing these same numerical 
methods on nonuniform meshes, such as those developed above, will likely prove to be a difficult task as discussed in St Cyr et al. [30]. While emerging numerical methods based on spectral elements, discontinuous Galerkin, finite-element or similar approaches are a more natural choice when considering the multi-resolution meshes developed here, these alternative approaches are still relatively new to climate system modeling in comparison to loworder finite volume methods. The sole purpose of this section is to exhibit a low-order finite volume method capable of producing robust simulations when implemented on nonuniform SCVTs. The implication is that these meshes are immediately applicable to current generation climate system model components. With this purpose in mind, the discussion below is not intended to be exhaustive. In many respects, developing numerical methods that effectively utilize these nonuniform SCVTs is a much richer and more difficult problem than generating the mesh itself. While some efforts to exploit the local uniformity of SCVTs have already been completed (e.g. see [6]), much work remains. Our progress on this will be reported in more detail at a later time.

\subsection{Continuous equations}

For this demonstration, we choose the nonlinear shallow water equations spanning the entire surface of the sphere:

$$
\begin{aligned}
& \frac{\partial h}{\partial t}+\nabla \cdot(h \underset{\sim}{u})=0 \\
& \frac{\partial \underset{\sim}{u}}{\partial t}+(\omega+f) \underset{\sim}{\underset{\sim}{x}} \underset{\sim}{u}=-g \nabla\left(h+h_{s}\right)-\frac{1}{2} \nabla\|\underset{\sim}{u}\|^{2} \\
& \omega=\underset{\sim}{k} \cdot(\nabla \times \underset{\sim}{u})
\end{aligned}
$$

where $h$ is the fluid thickness, $h_{s}$ is the height of the lower boundary, $\underset{\sim}{u}$ is the vector velocity orthogonal to the local normal vector $\underset{\sim}{k}$ and $f$ is the Coriolis parameter. The component of relative vorticity in the plane normal to the surface of the sphere, $\omega$, defined in (11)

\subsection{Discrete equations}

We utilize the discrete method developed by Bonaventura and Ringler [4]. While the method developed in [4] is intended for use on multi-resolution meshes, to our knowledge this is the first demonstration. This method uses the Delaunay triangulation as the finite-volume cell for the thickness equation. The vorticity field is defined on the Voronoi diagram. Velocity components normal to the triangle edges are retained as prognostic equations. A schematic of this discretization is shown in Figure 14. All quantities with overhats are derived fields with $\hat{T}$ representing the reconstructed tangent velocity required for the Coriolis force and $\hat{\eta}$ representing the absolute vorticity (see [4] for a full discussion). The discrete system is expressed as:

$$
\frac{\partial h_{i}}{\partial t}=\frac{-1}{A_{i}} \sum_{j=1}^{\text {nedges }} \hat{h}_{j} N_{j} d l_{j}
$$




$$
\frac{\partial N_{j}}{\partial t}=\hat{\eta}_{j} \hat{T}_{j}-\left\{\left[g h+g h_{s}+\hat{K}\right]_{i F o r w a r d}-\left[g h+g h_{s}+\hat{K}\right]_{i B a c k w a r d}\right\} / d c_{j}
$$

where the summation in (12) is over the edges of each triangle. In terms of solution error, the scheme is nominally second-order accurate in space using centered-in-space reconstructions and fourth-order accurate in time using 4th-order Runge-Kutta time-stepping (see e.g. [4]). The simulations utilize no limiters, filters or explicit dissipation of any sort. The centerin-space numerics, along with the 4th order Runge-Kutta scheme, is used to minimize any implicit diffusion.

We demonstrate this method on two SCVTs shown in Figure 15. Each mesh contains 40962 nodes. The solid black line indicates the boundary of an orographic feature that is the sole forcing of the simulation (see below). The first mesh (top) is generated with a uniform density function leading to an average grid spacing of $120 \mathrm{~km}$. The second mesh (bottom) is generated with higher densities in the vicinity of the orographic feature. The density function is chosen such that the average grid spacing is three time smaller $(40 \mathrm{~km})$ in the vicinity of the mountain than compared to its quasi-uniform counterpart. The solid colors indicate our domain-decomposition strategy for efficient implementation on distributed memory systems: each color represents a different computational processor.

\subsection{Simulation}

We apply this numerical method to one of the standard shallow-water test cases developed by Williamson et al. [33] referred to as Test Case 5. In this test case a flow in geostrophic balance is confronted with a large-scale orographic feature at the start of the simulation, $\mathrm{t}=0$. The transient forcing at $\mathrm{t}=0$ leads to the generation of large amplitude gravity waves and Rossby waves. The sole forcing mechanism is the presence of the orographic forcing. While no analytical solution is known, results from high resolution global spectral models are adequate reference solutions for the simulations conducted here (e.g. see [17]).

Both simulations are stable over the course of the 15 day integration. The kinetic energy field for each simulation is shown in Figure 16. Both simulation produce the same largescale flow structure: an anticyclone dominates in the region of orography with a strong, stationary low-pressure system residing immediately downstream. Both simulations produce velocities in excess of $40 \mathrm{~m} / \mathrm{s}$ in the jet region.

Figure 17 shows how the error norms for each of these simulations evolve over the course of the simulation. The error is based on the deviation of the thickness field from the high-resolution spectral results. The panel on the left shows the normalized $L_{2}$-error following the procedure in [31] Eq. 17. The $L_{2}$ error norm is computed for two regions: a global domain and a local domain defined by $h_{s}>0$ that is coincident to the region of enhanced resolution. The panel on the bottom depicts the $L_{\infty}$-norm with the same layout. Since the $L_{2}$ error is normalized by the reference values, we only compare norms within the same averaging domain. When comparing the global $L_{2}$ error norms between the simulations, we find that the variable-resolution mesh provides marginal improvements only for times less than 24 hours. At the early stages of the simulation, the benefit of the variable-resolution mesh is due mostly to a better representation of the initial condition. For the remainder of the integration, the two simulations have nearly identical global $L_{2}$ error values. When we compare the local $L_{2}$ error values, we find a slightly different result; averaged over the duration of the simulation the variable-resolution mesh reduces the error by approximately 20 percent. The $L_{\infty}$ error values (right panel) exhibit a similar tendency. The variable-resolution mesh 
provides little benefit in the context of global error reduction, but does significantly reduce the errors in the vicinity of the orography. In this case, the variable-resolution mesh reduces the local $L_{\infty}$ error norm by a factor of two as compared to the uniform mesh simulation. The implications of these findings on the merit of multi-resolution climate system models will be discussed in the next section.

\section{Discussion and Conclusions}

We have argued that the traditional paradigm of constructing IPCC-class climate models based on quasi-uniform meshes will be strained in the coming decade by two mechanisms. First, each climate system model component currently has one or more unresolved processes that may play an important role in the dynamics of the global climate system. These processes are either omitted altogether, exemplified by the omission of ice streams in ice sheet models, or highly parameterized, exemplified by the parameterization of eddy activity in the ocean. The current and foreseeable computational resources preclude the notion of resolving these processes everywhere all of the time. Second, IPCC-class climate models will be pressed into the role of simulating regional climate change with the purpose of developing adaptation strategies. The resolution and computational resources required for the robust simulation of regional climate change will force the climate modeling community to develop an alternative approach to compliment the current suite of quasi-uniform global climate system models.

One promising approach to meet these new challenges is based on the use of Spherical Centroidal Voronoi Tessellations. These tessellations (or meshes) offer many attractive qualities in the context of climate system modeling. First, since these meshes are a superset of the commonly used "icosahedral-hexagonal grids," we can conceptually consider SCVTs to be an extension of meshes already in use today. Second, SCVTs allow for the spatial allocation of nodes in an straight-forward, intuitive manner. SCVTs are generated with respect to a user-defined density function where nodes are "clustered" toward regions of high density and away from regions of low density. If we understand a system well enough to know how to redistribute our degrees of freedom (and hence our computational resources), SCVTs offer an easy way to implement this redistribution. Finally, and most importantly, SCVTs are amenable to rigorous analysis from which we can make statements regarding the regularity of a given mesh and how that regularity will improve as we increase the nodes in a given domain.

We demonstrated the potential for this technique by developing example meshes for several different components of the Earth's climate system: the Greenland ice sheet, the North Atlantic ocean and a generic Antarctica ice shelf-ocean interaction. Furthermore, our example numerical method developed a multi-resolution mesh that is characteristic of local resolution enhancement in regional atmospheric modeling. In each of these examples we exhibited the ability to precisely manipulate the regions of enhanced resolution through our choice of the SCVT density function. In two of the examples (Greenland and North Atlantic) the SCVT density function was developed directly from physical characteristics of the system. In the Greenland example, we used the observed ice thickness distribution to develop a SCVT density function that places increased resolution at the ice margins. In the North Atlantic example, we constructed the SCVT density function in order to obtain meshes that are able to resolve eddy activity associated with the Gulf Stream and North Atlantic current. In each of these examples we computed metrics that measure the quality of the mesh. In all cases, and in agreement with the theoretical underpinnings of SCVT, we 
found that increasing the degrees of freedom results in an uniform improvement in mesh quality. We found this consistent improvement in both the Voronoi diagrams as well as in the dual Delaunay triangulations.

While the primary purpose of this work is to demonstrate the potential for SCVTs to produce high-quality, multi-resolution meshes for climate system applications, we felt it important to also exhibit a traditional, finite-volume technique that can successfully exploit the benefits of a variable-resolution mesh. While our results in this regard are far from sufficient, we have at least produced one positive example in the context of the global shallow-water equations. Even this simple example has provided some guidance on what we should and should not expect from multi-resolution techniques such as the one developed here. For instance, given the hyperbolic nature of many of the Earth's climate system components it will be extremely difficult to reduce formal solution error over a wide range of conditions. Eventually, the error will become dominated by some phenomena (transient or otherwise) occurring in regions of low resolution. While this problem occurs regardless of the numerical method employed, it will likely be particularly evident when using the low-order finite volume methods that are ubiquitous in climate system models today. In contrast to reducing formal solution error, our emphasis will be on the formulation of robust numerical methods that produce stable, long-time simulations over a wide class of phenomena without the need for $a d$ hoc filtering or dissipation. The driving purpose for developing multi-resolution climate system components will be for the simulation of new phenomena requiring enhanced resolution, not necessarily for the formal reduction in solution error.

While this work has demonstrated the ability to generate high-quality meshes for a wide class of problems, the daunting challenge going forward to is develop numerical techniques that can effectively exploit these high-quality, multi-resolution meshes.

Acknowledgements This work was supported by the DOE Office of Science's Climate Change Prediction Program through DE-FG02-07ER64431, DE-FG02-07ER64432 and DOE 07SCPF152.

\section{References}

1. Climate Change 2007: The SCIEntific Basis, International Panel on Climate Change, 2007

2. BAmber, J.L., BALDwin, D.J., Gogineni, S.P., A new bed elevation data set for modelling the Greenland ice sheet, Ann. Glaciol., 37, pp. 351-356, (2003).

3. RobIN E. BELL, The role of subglacial water in ice-sheet mass balance Nature Geosciences, 1, pp 297304, (2008)

4. Bonaventura, L., And T. Ringler, Analysis of Discrete Shallow-Water Models on Geodesic Delaunay Grids with C-Type Staggering, Mon. Wea. Rev., 133, pp. 23512373, (2005).

5. Collins, W.D., C.M. Bitz, M.L. Blackmon, G.B. Bonan, C.S. Bretherton, J.A. Carton, P. Chang, S.C. Doney, J.J. Hack, T.B. Henderson, J.T. Kiehl, W.G. Large, D.S. McKenna, B.D. SANTER, AND R.D. SMITH,The Community Climate System Model Version 3 (CCSM3), J. Climate, 19, pp. 21222143, (2006).

6. Q. DU AND L. JU, Finite volume methods on spheres and spherical centroidal Voronoi meshes, SIAM Journal on Numerical Analysis, 43, pp. 1673-1692, (2005).

7. Q. DU, V. FABER, AND M. GunZBURGer, Centroidal Voronoi tessellations: Applications and algorithms, SIAM Review, 41, pp. 637-676, (1999).

8. Q. DU, M. GunZBURGER, AND L. JU, Constrained centroidal Voronoio tessellations on general surfaces, SIAM J. Sci. Comput. 24, pp. 1488-1506, (2003).

9. Q. Du, M. GunzBurger, And L. Ju, Voronoi-based finite volume methods, optimal Voronoi meshes and PDEs on the sphere, Comput. Meths. Appl. Mech. Engrg. 192, pp. 3933-3957, (2003).

10. Q. DU AND D. WANG, Tetrahedral mesh generation and optimization based on centroidal Voronoi tessellations, Int. J. Numer. Methods Engrg. 56, pp. 1355-1373. (2003).

11. D. FIELD, Quantitative measures for initial meshes, Int. J. Numer. Methods Engrg. 47, pp. 887-906, (2000). 
12. A. Gersho AND R. GRAY, Vector Quantization and Signal Compression, Kluwer, Boston, (1992).

13. Hallberg, R., And A. Gnanadesikan, The Role of Eddies in Determining the Structure and Response of the Wind-Driven Southern Hemisphere Overturning: Results from the Modeling Eddies in the Southern Ocean (MESO) Project J. Phys. Oceanogr., 36, pp. 2232-2252, (2006).

14. Holland, P.R., A Jenkins, AND D.M Holland The response of ice shelf basal melting to variation in ocean temperature, $J$ Climate, in press.

15. Joughin, I., L. Gray, R. Bindschadler, S. Price, D. Morse, C. Hulbe, K. Mattar and C. WERNER, Tributaries of West Antarctic Ice Streams Revealed by RADARSAT interferometry, Science, 286(5438), pp. 283-286, (1999).

16. L. JU, M. GUNZBURGER AND W.-D. ZHAO, Adaptive finite element methods for elliptic PDEs based on conforming centroidal Voronoi Delaunay triangulations, SIAM J. Sci. Comput. 28, pp. 2023-2053, (2006).

17. LipsCOMB, W.H., AND T.D. RingleR, An Incremental Remapping Transport Scheme on a Spherical Geodesic Grid. Mon. Wea. Rev., 133, pp. 23352350 (2005)

18. S. LLOYD, Least squares quantization in PCM, IEEE Trans. Infor. Theory 28, pp. 129-137. (1982).

19. J. MacQueEn, Some methods for classification and analysis of multivariate observations, Proc. Fifth Berkeley Symposium on Mathematical Statistics and Probability, Vol I, University of California, pp. 281297, (1967).

20. McGregor J. L., Semi-Lagrangian advection on a conformal cubic grid. Mon. Wea. Rev., 124, pp. 13111322, (1996).

21. M.E. Maltrud And J.L. MCClean, An eddy-resolving global 1/10 degree ocean simulation, Ocean Model. 8, pp. 3154, (2004)

22. Newman, B.D., Wilcox, B.P., Archer, S., Breshears, D.D., Dahm, C.N., Duffy, C.J., McDowell, N.G., Phillips, F.M., Scanlon, B.R. And Vivoni, E.R., The Ecohydrology of Arid and Semiarid Environments: A Scientific Vision, Water Resources Research, 42, W06302, doi:10.1029/2005WR004141, (2006).

23. A. Okabe, B. Boots, K. Sugihara, And S. Chiu, Spatial Tessellations: Concepts and Applications of Voronoi Diagrams, 2nd edition, Wiley, Chichester, (2000).

24. R. RENKA, ALGORITHM 772. STRIPACK: Delaunay triangulation and Voronoi diagrams on the surface of a sphere, ACM Trans. Math. Soft. 23, pp. 416-434, (1999).

25. ERIC Rignot, Jonathan L. BAmber, Michiel R. VAN DEN BRoEKe, CuRT Davis, Yonghong Li, WiLlem JAN VAN DE BERG AND ERIK VAN MEIJGAARD, Recent Antarctic ice mass loss from radar interferometry and regional climate modelling, Nature Geoscience, 1, pp. 106 - 110, (2008),

26. Rignot, E., G. Casassa, P. Gogineni, W. Krabill, A. Rivera and R. Thomas. Accelerated ice discharge from the Antarctic Peninsula following the collapse of Larsen B ice shelf. Geophys. Res. Lett., 31 (18), L18401. (10.1029/2004GL020697), (2004).

27. Ringler, T.D., R.P. Heikes, And D.A. Randall, Modeling the Atmospheric General Circulation Using a Spherical Geodesic Grid: A New Class of Dynamical Cores. Mon. Wea. Rev., 128, pp. 24712490, (2000).

28. Smith, R.D., M.E. Maltrud, F.O. Bryan, and M.W. Hecht, Numerical Simulation of the North Atlantic Ocean at 1/10. J. Phys. Oceanogr., 2000, 30, pp. 15321561, (2000).

29. Schoof,C., Ice sheet grounding line dynamics: steady states, stability, and hysteresis. Journal of Geophysical Research, 112(F3), F03S28, (2007).

30. St-Cyr, A., C. Jablonowski, J. M. Dennis, H. M. Tufo and S. J. Thomas, A Comparison of Two Shallow Water Models with Non-Conforming Adaptive Grids, Mon. Wea. Rev., 136, pp. 1898-1922, (2007),

31. Tomita, H, M. Tsugawa, M. SAtoh, And K. Goto, Shallow Water Model on a Modified Icosahedral Geodesic Grid by Using Spring Dynamics Journal of Computational Physics, 174, pp. 579613, (2001)

32. Tomita, H., H. Miura, S. Iga, T. NAsuno, And M. Satoh, A global cloud-resolving simulation: Preliminary results from an aqua planet experiment, Geophys. Res. Lett., 32, L08805, doi 10.1029/2005GL022459, (2007).

33. Williamson D. L., J. B. Drake, J. Hack, R. Jacob, and P. N. Swartztrauber. A standard test for numerical approximation to the shallow water equations in spherical geometry. J. Comput. Phys, 102, pp. 211224, (1992). 

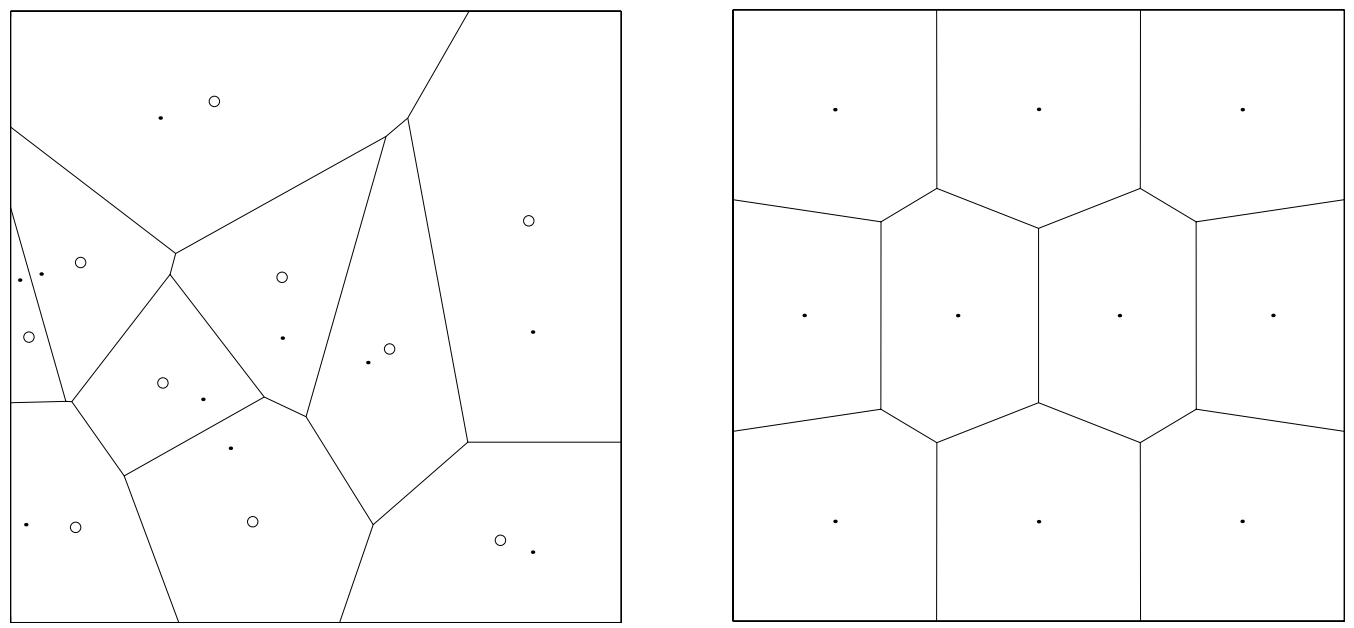

Fig. 1: Left: a Voronoi tessellation of a square in $\mathbb{R}^{2}$ with 10 generators (the dots) randomly selected (the circles denote the centroids); Right: a 10-point CVT with a constant density function of a square in $\mathbb{R}^{2}$. 

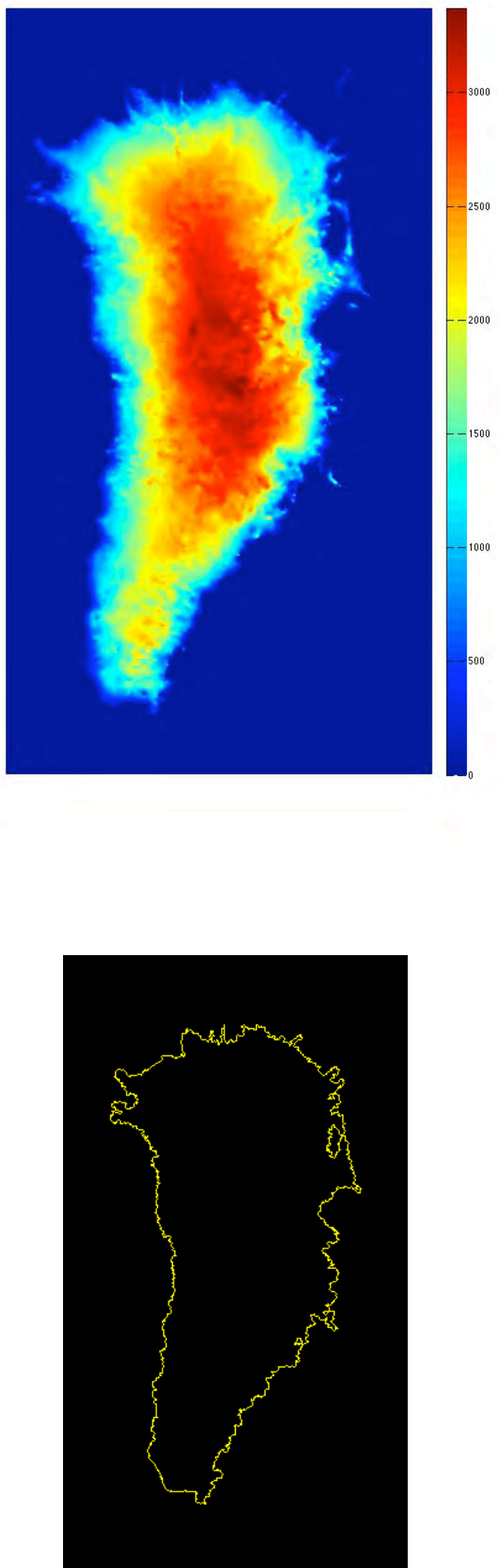

Fig. 2: Top: Distribution of Greenland ice thickness (meters) from [2]; Bottom: A piece-wise linear representation of the ice boundary obtained from the ice thickness distribution map. 


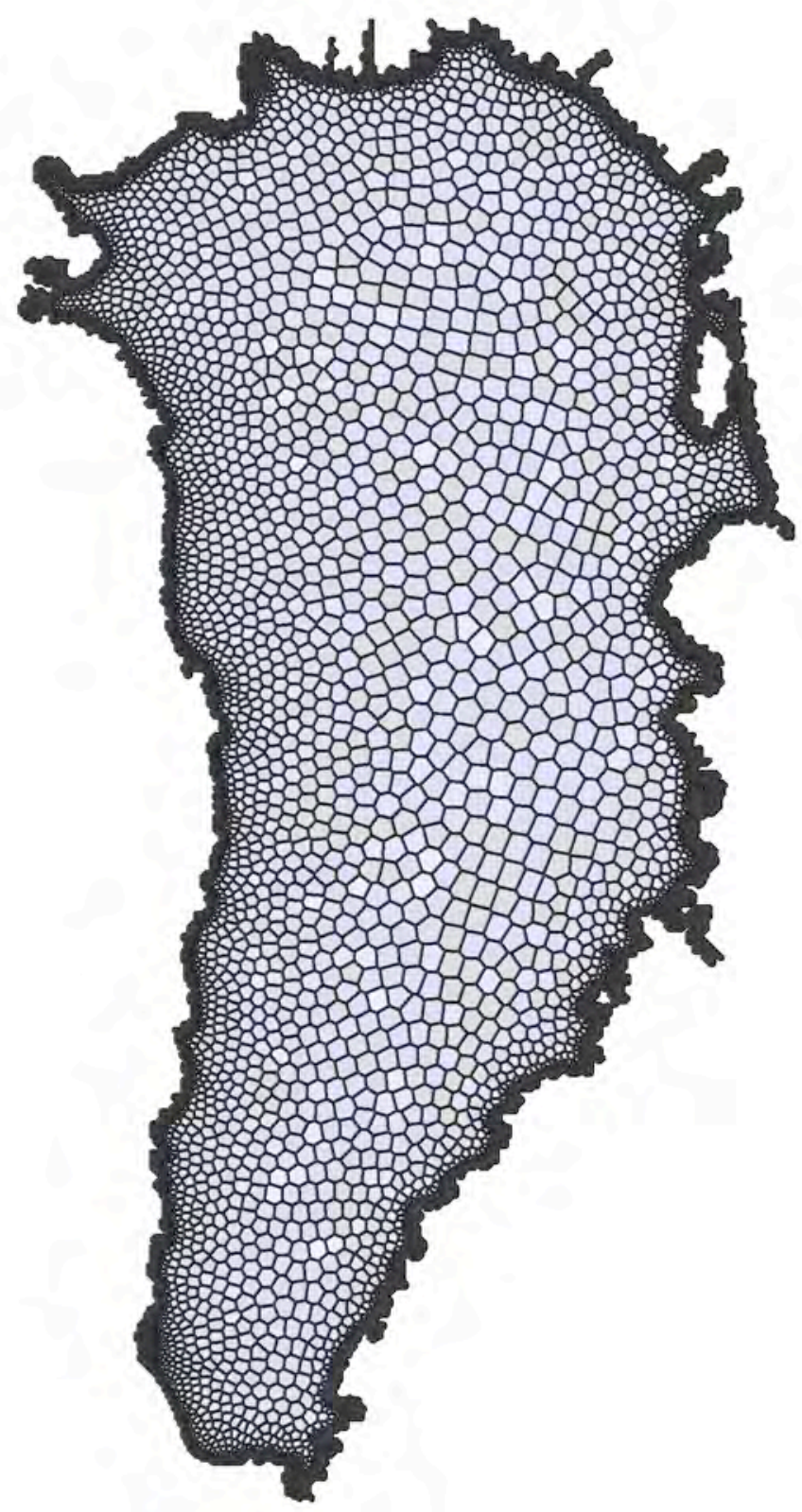

Fig. 3: A SCVT of the Greenland ice sheet using 29474 nodes based on the density function given in (7). Note that grid cells near the ice margin are too small to be visible 

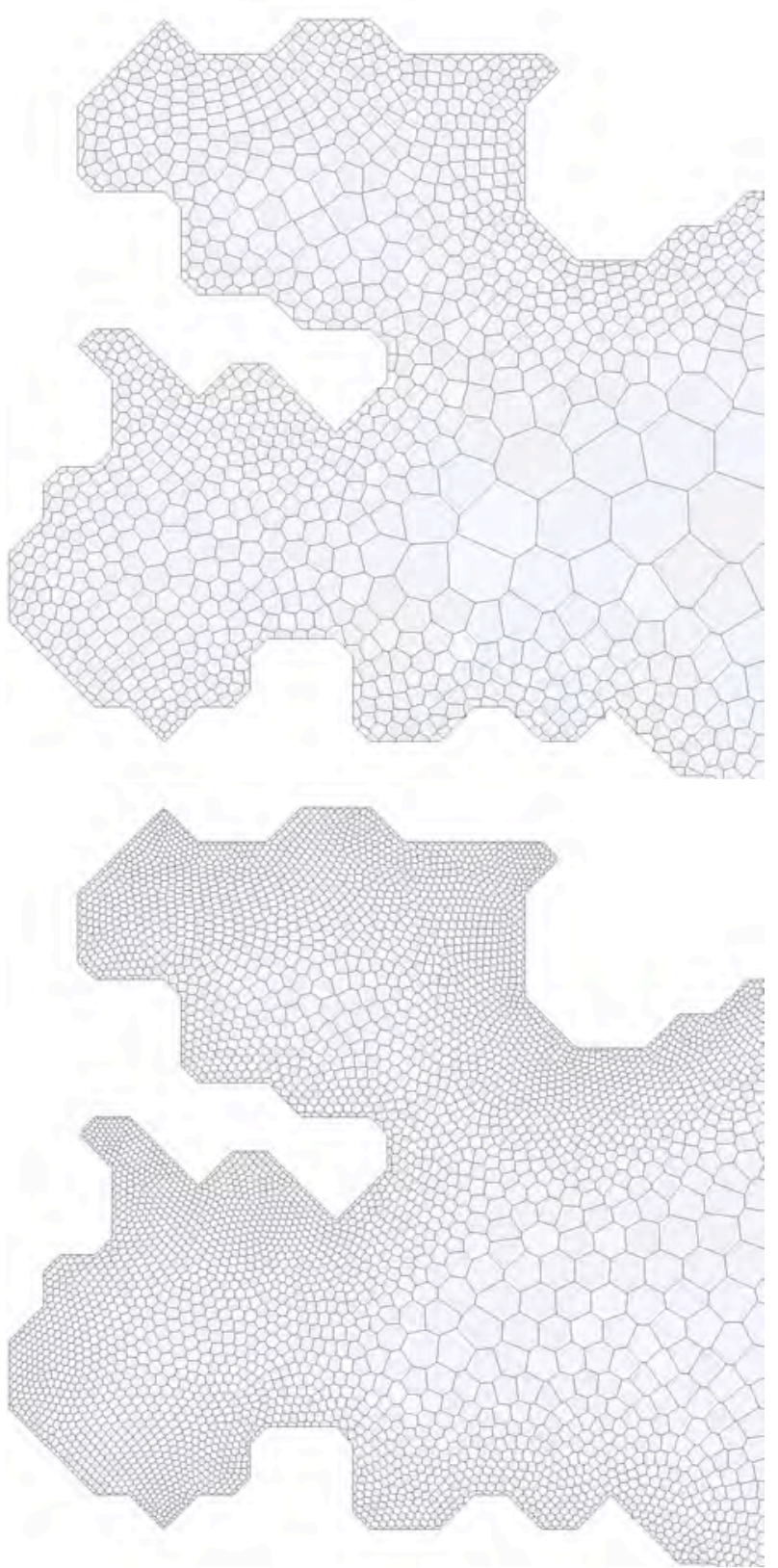

Fig. 4: A close-up on one region within the Greenland SCVT. Top: the SCVT using 29474 generators. Bottom: the SCVT using 112896 generators 

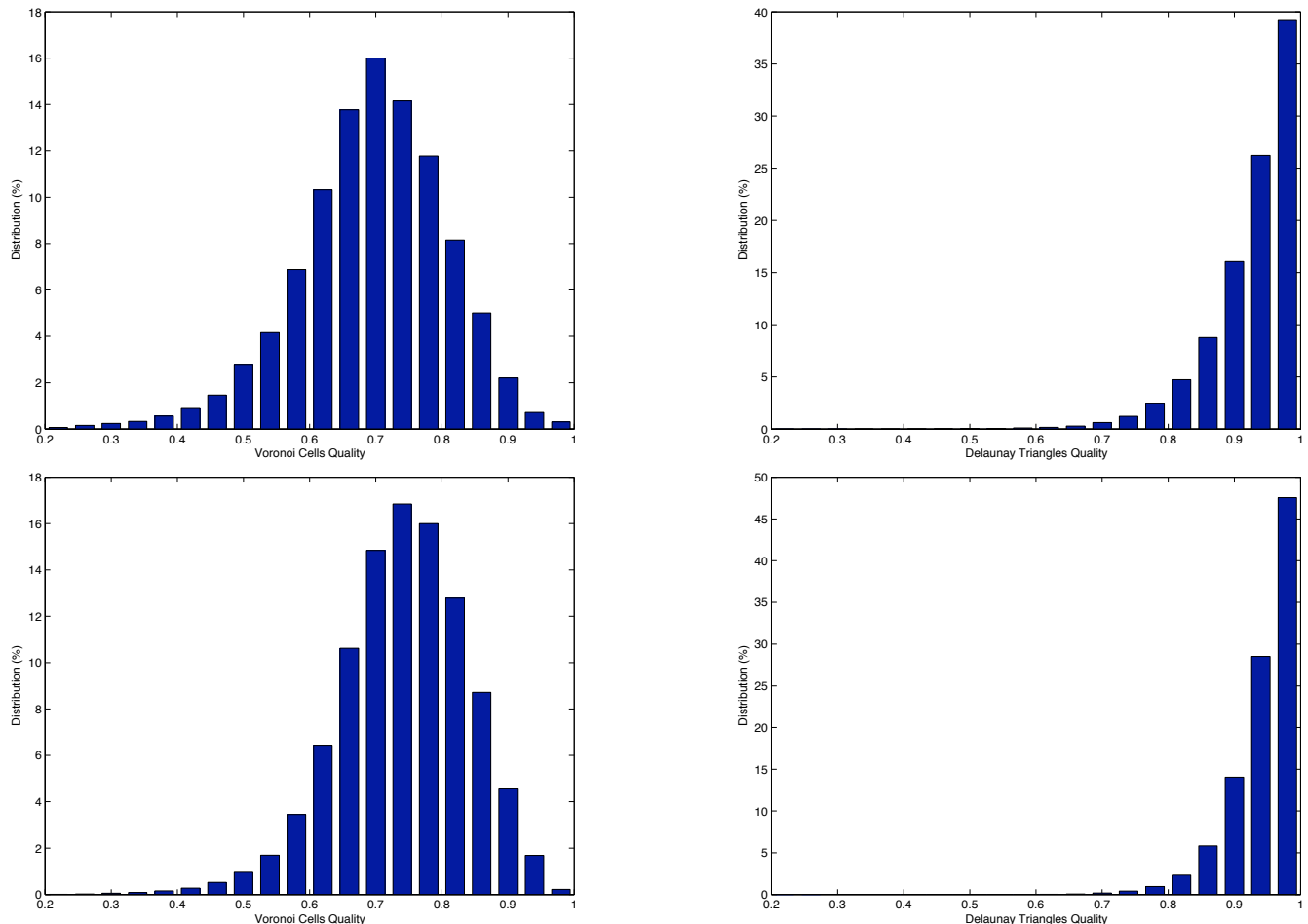

Fig. 5: Quality histograms of SCVTs of the Greenland ice sheet with 29474 nodes (top) and 112896 nodes (bottom). Left: Distribution of quality measurement of Voronoi cells $\sigma$; Right: Distribution of quality measurement of Delaunay triangles $q$.

Note the uniform shift toward higher-quality as the number of nodes is increased. 


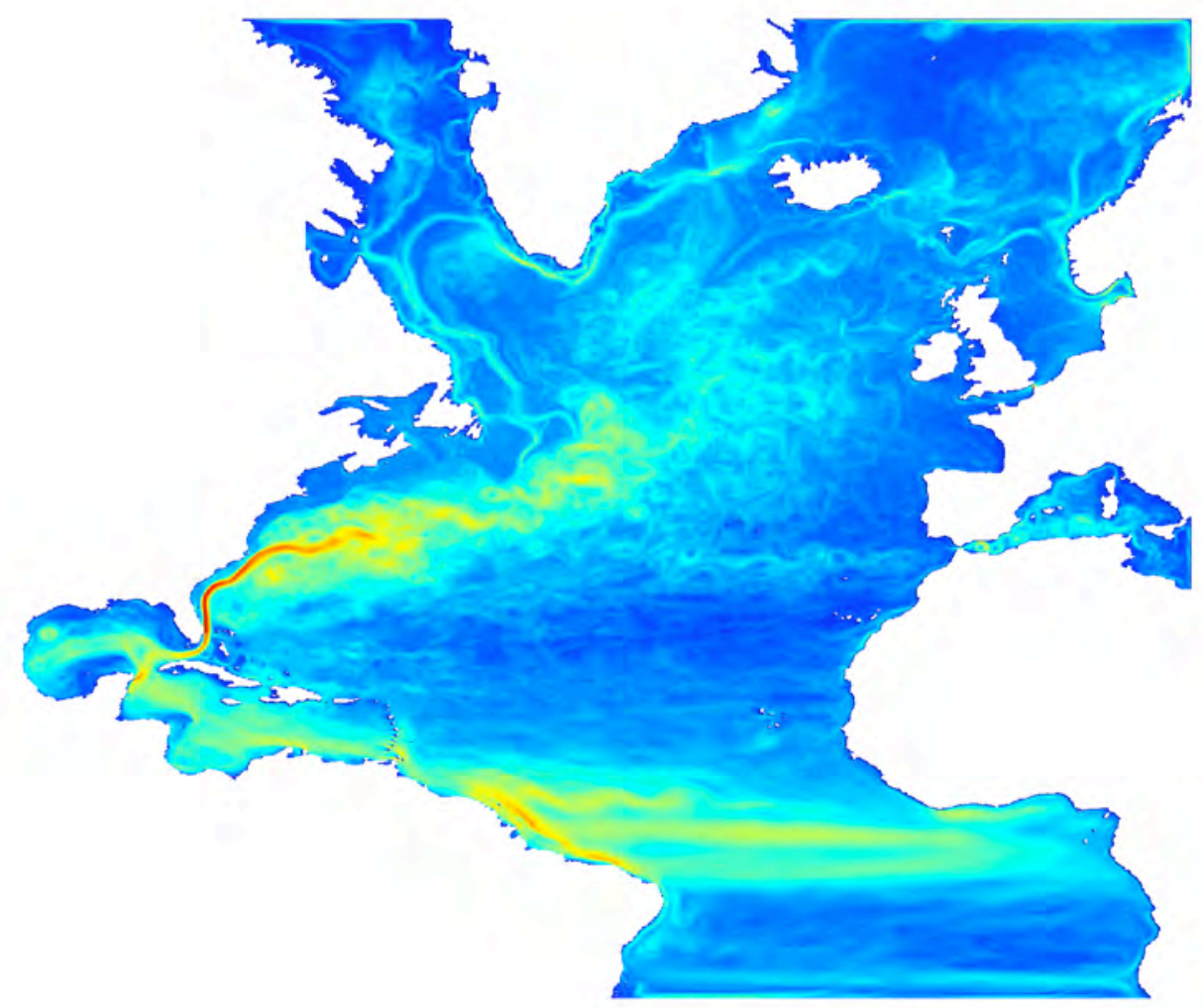

Fig. 6: Time mean kinetic energy from a global 0.1 degree simulation of the North Atlantic Ocean [28] 


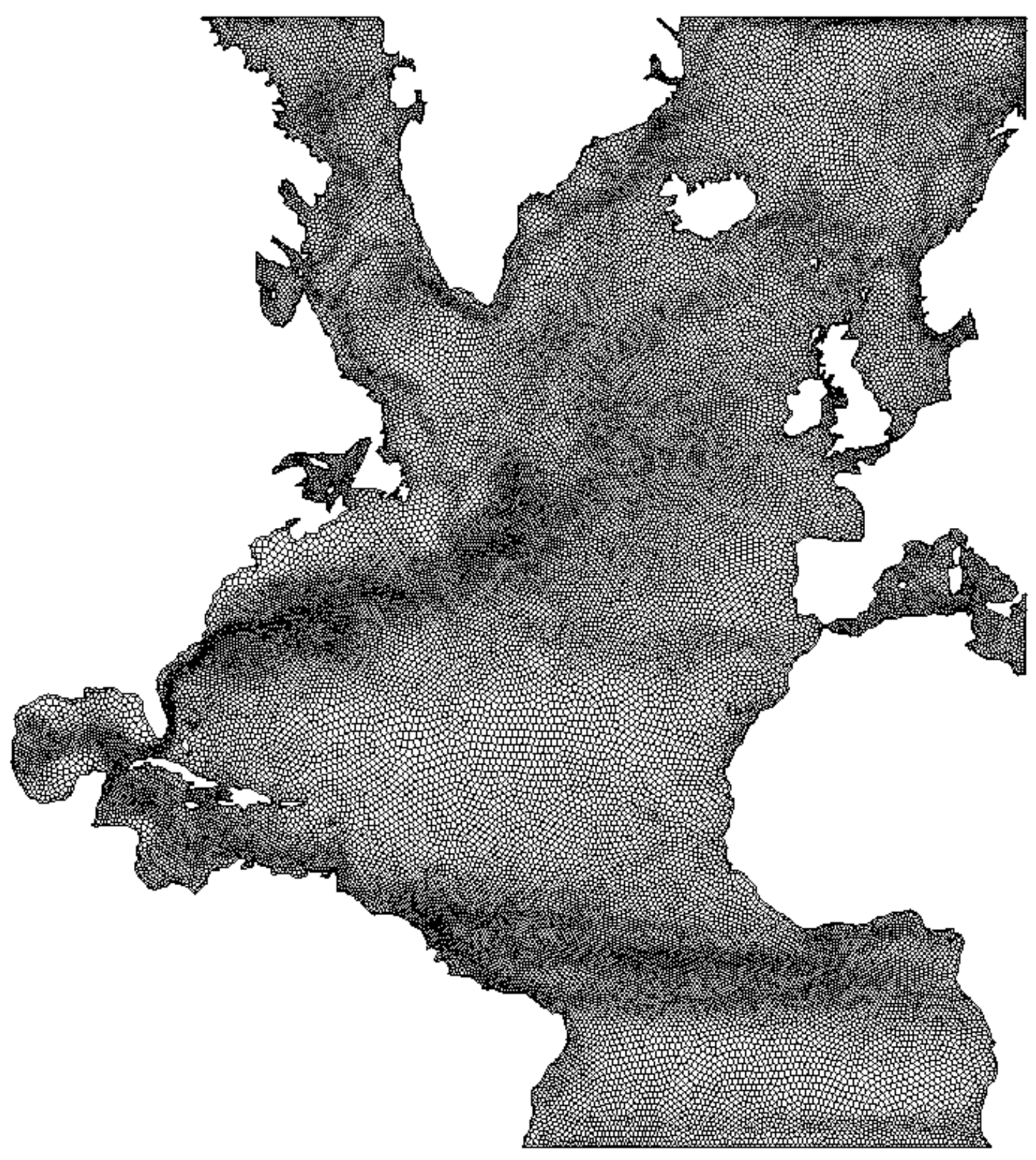

Fig. 7: SCVT of North Atlantic ocean domain using 40162 nodes. 

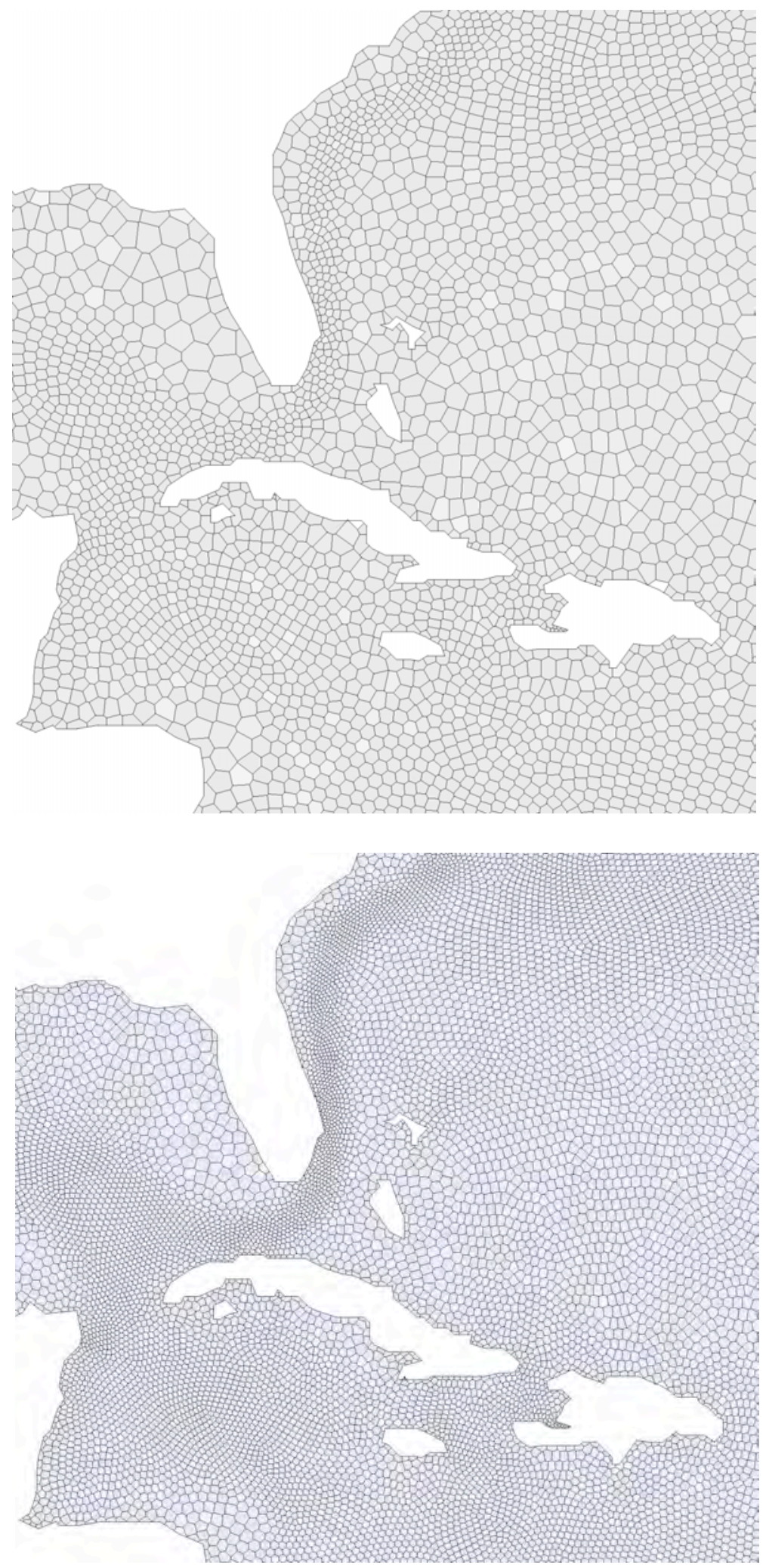

Fig. 8: Close-up of Gulf Stream region using 40162 nodes (top) and 157366 nodes (bottom) 

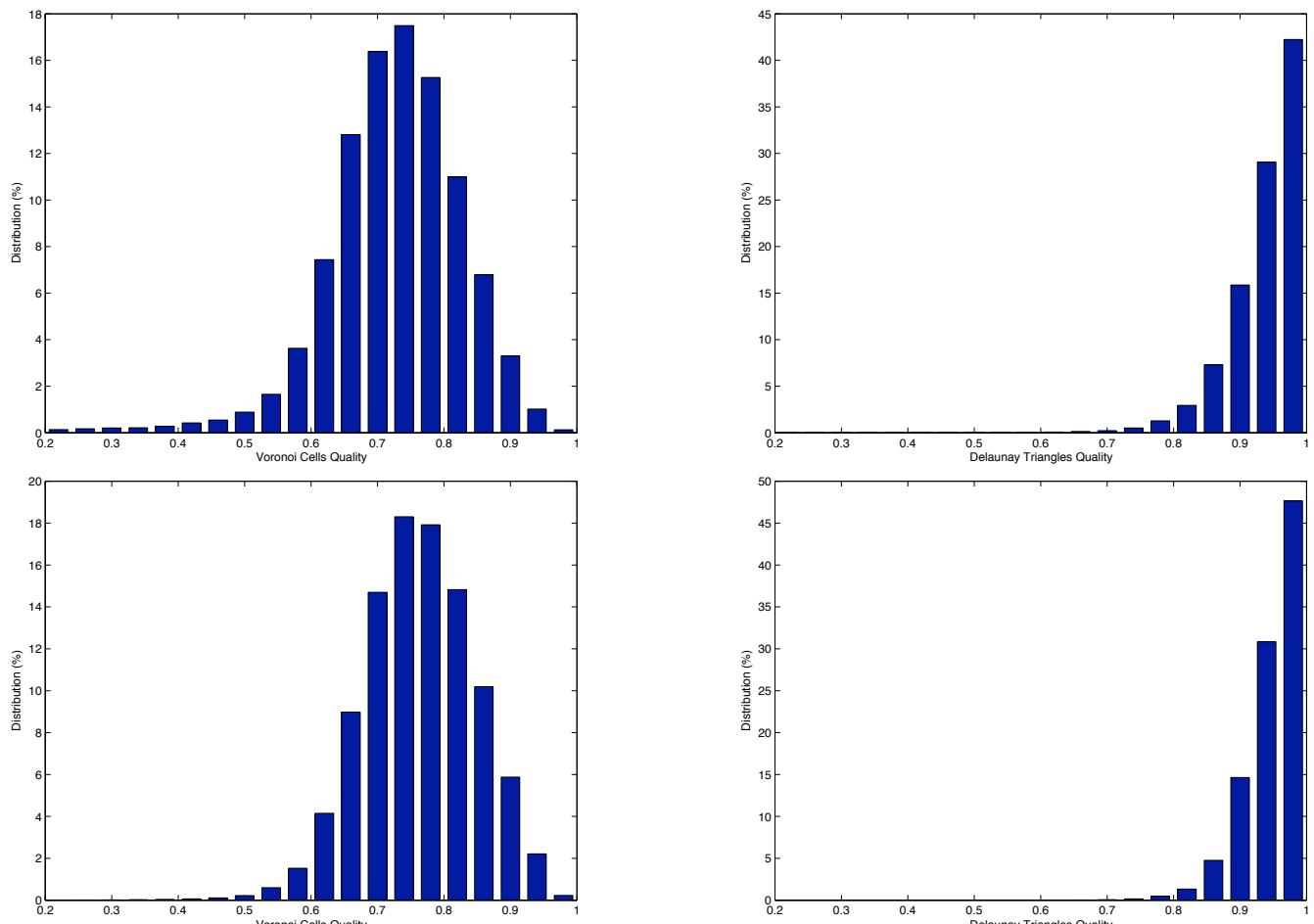

Fig. 9: Quality histograms of SCVTs of the North Atlantic with 40162 nodes (top) and 157366 nodes (bottom). Left: Distribution of quality measurement of Voronoi cells $\sigma$; Right: Distribution of quality measurement of Delaunay triangles $q$. 


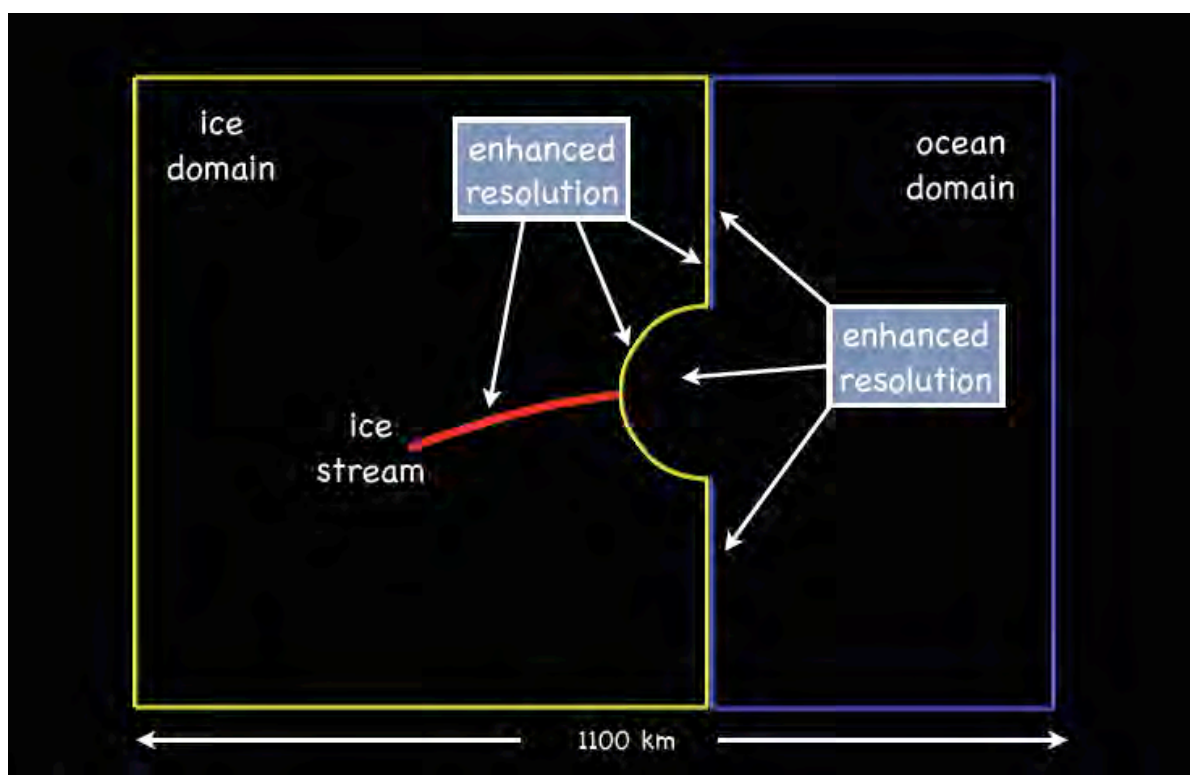

Fig. 10: An idealized ocean-ice shelf system. The ice domain (left) flows into the shelf region (semi-circle) via an ice stream. Enhanced resolution in the vicinity of the ice stream, ice shelf and ice margin will be required. 


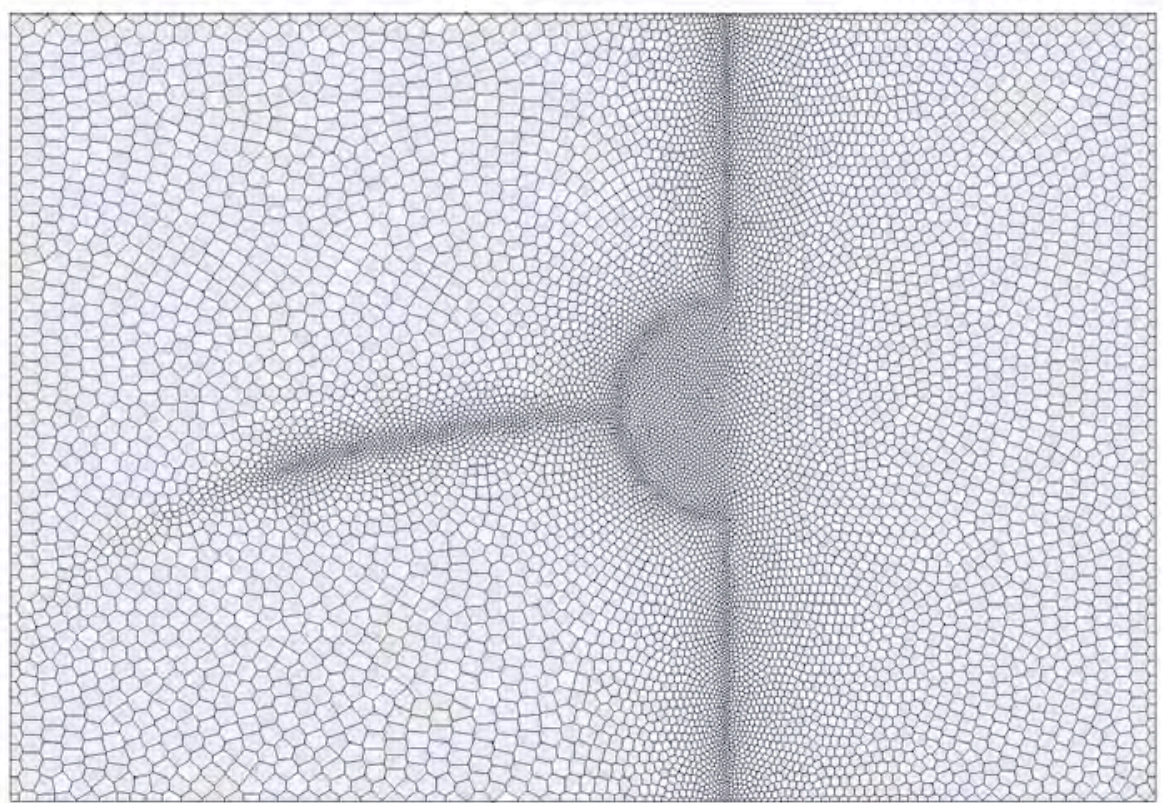

Fig. 11: SCVT of ocean-ice shelf system using 9359 nodes. Note enhanced resolution in the vicinity of the ice stream, ice shelf and ocean-ice interface.
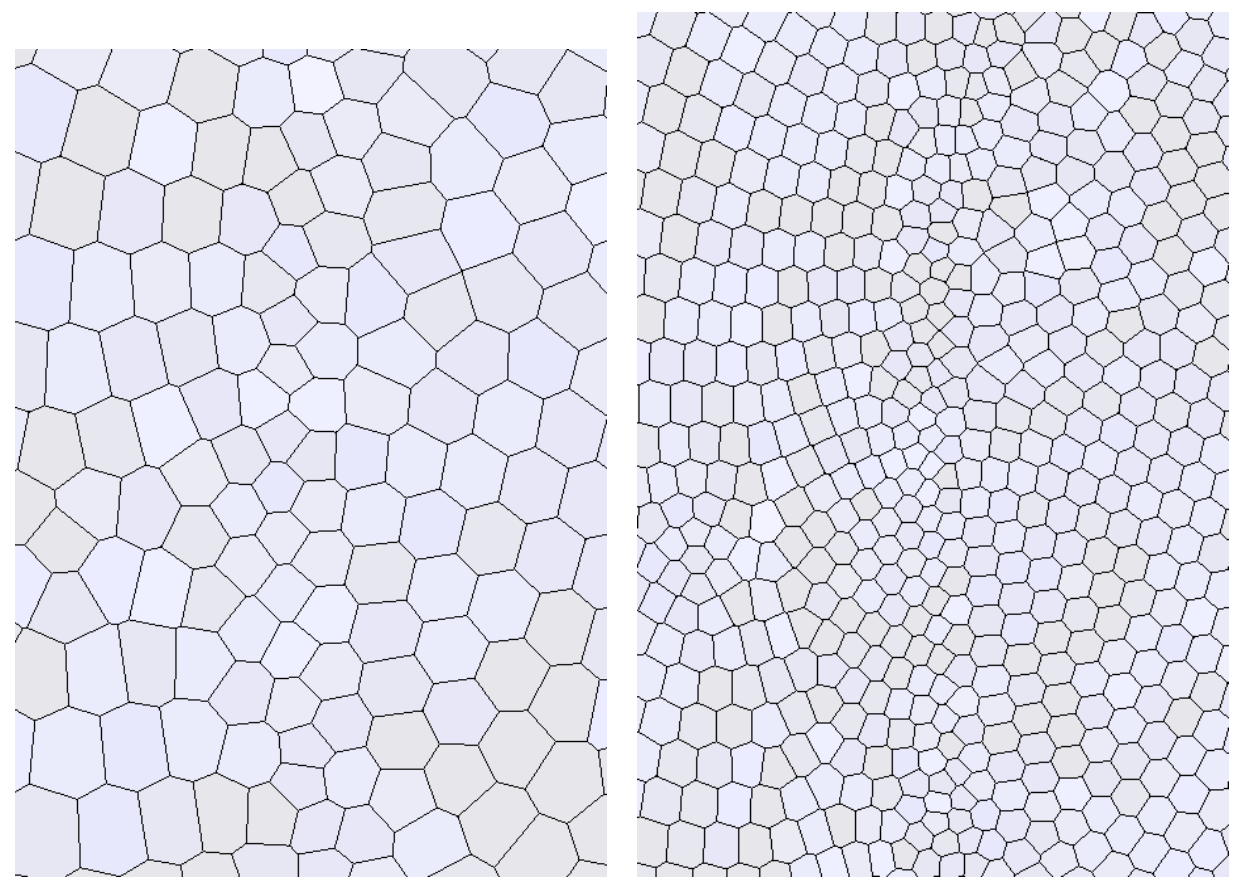

Fig. 12: Close-up of ocean-ice shelf SCVT in the vicinity where the ice stream enters the shelf region. Left: SCVT using 9359 nodes. Right: SCVT using 37157 nodes. 

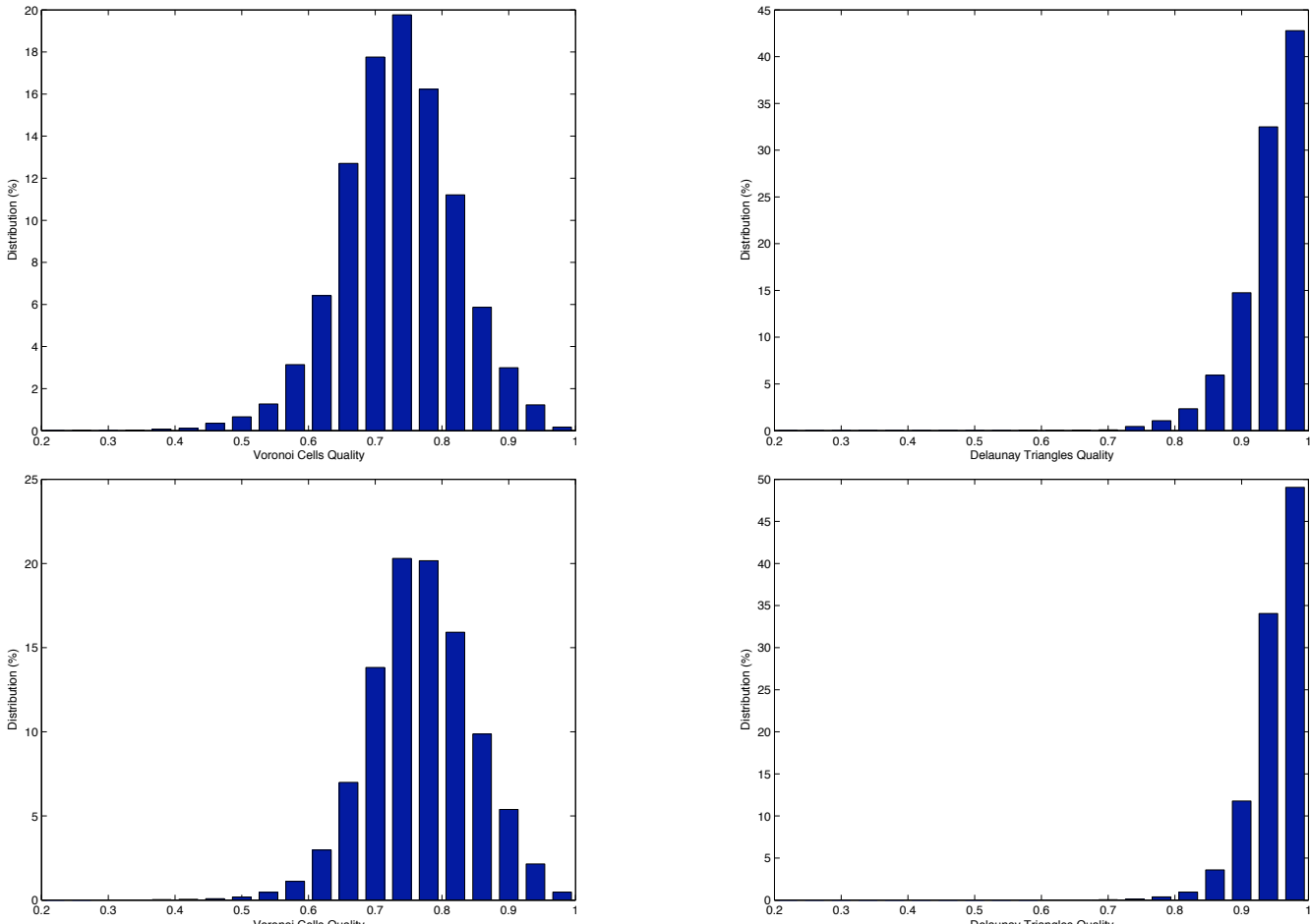

Fig. 13: Quality histograms of SCVTs of the idealized ocean-ice sheet with 9359 nodes (top) and 37157 nodes (bottom). Left: Distribution of quality measurement of Voronoi cells $\sigma$; Right: Distribution of quality measurement of Delaunay triangles $q$. 


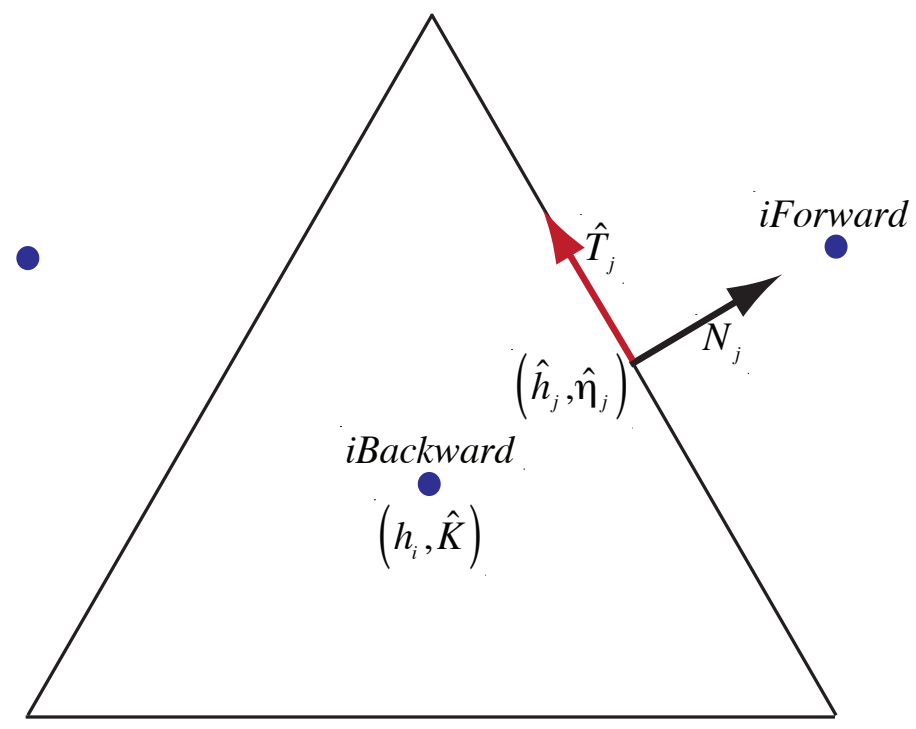

Fig. 14: A schematic of the finite-volume system. Thickness, $h$, and kinetic energy, $\hat{K}$, are defined at the centers of the triangles. The normal component of velocity, $N_{j}$ is defined at each cell edge. Vorticity, $\eta$, is defined at the triangle vertices. All quantities with overhats are derived fields, see [4] for details. 

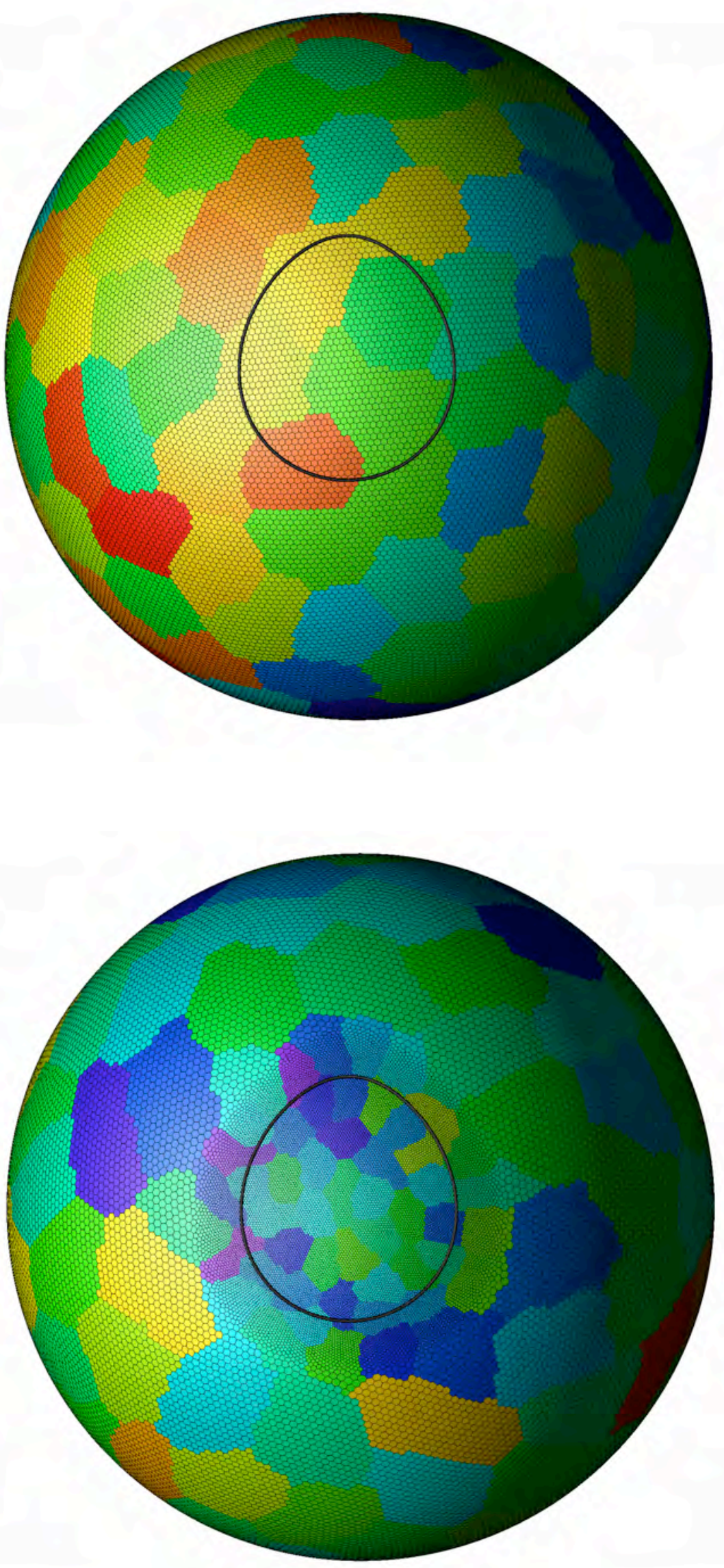

Fig. 15: Left: A SCVT using 40962 nodes with a uniform density function. Right: A SCVT also using 40962 nodes but using a nonuniform density function with high values of density occurring in the vicinity of the orography (shown by solid black line). The variable-resolution mesh results in nominal grids spacing of approximately 1/3 that found in the quasi-uniform mesh. The colored background denotes groups of cells (blocks) that are distributed across multiple processors. 

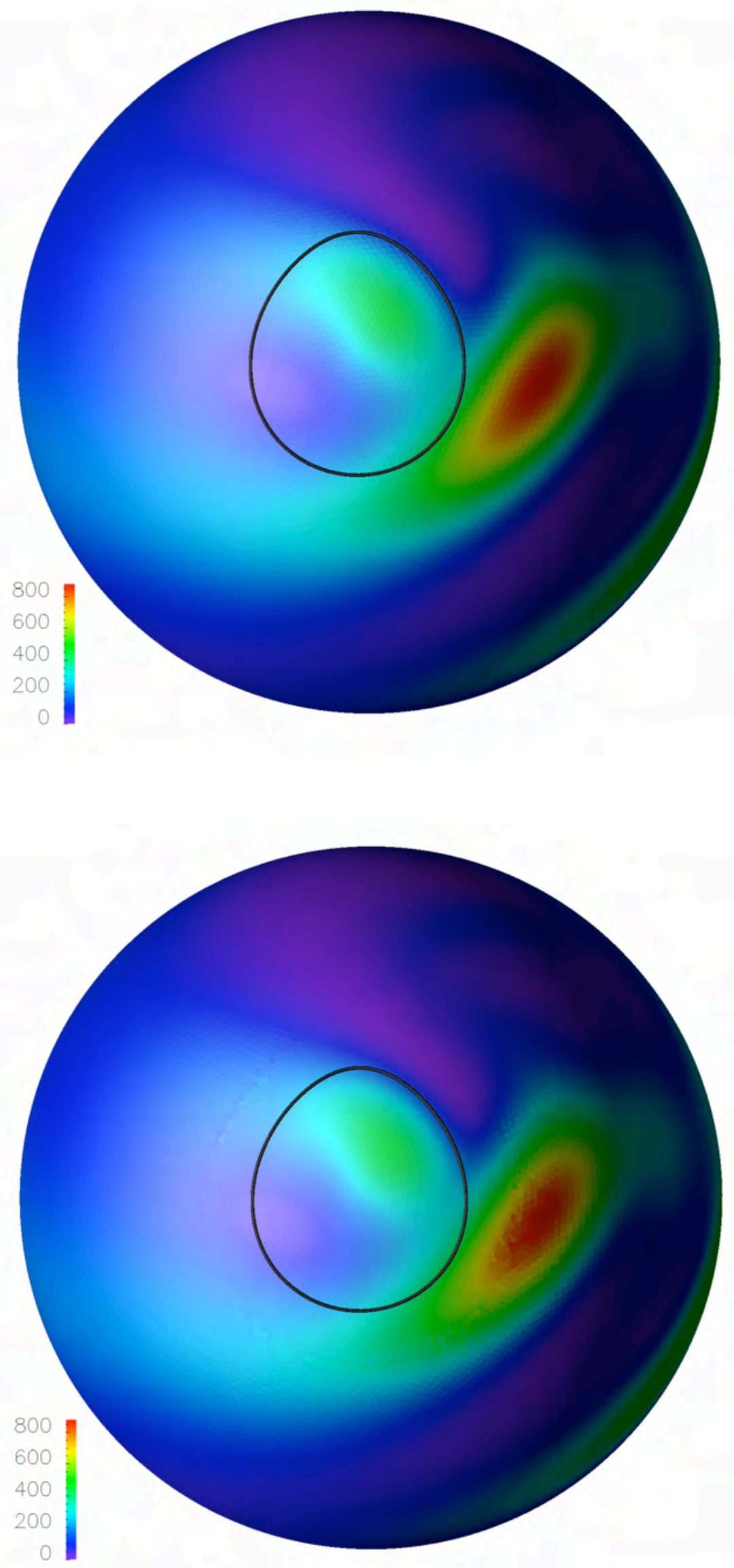

Fig. 16: Kinetic energy field at day 10 of simulation. Right: Simulation using quasi-uniform mesh. Left: Simulation using variable-resolution mesh. 

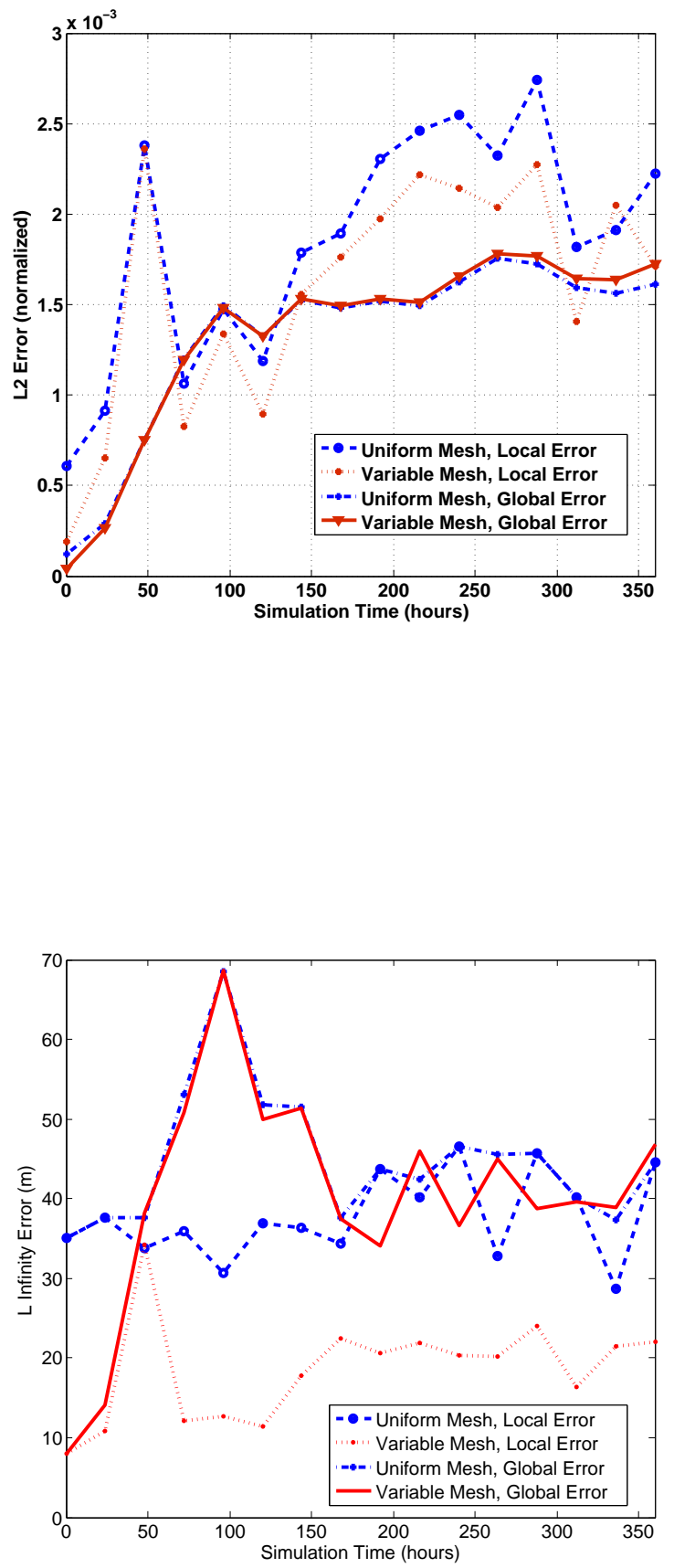

Fig. 17: Left: $L_{2}$ error norms for both the global domain and the local domain in the vicinity of the orography. Right: $L_{\infty}$ norms for the same two domains. Each figure compares the errors produced in the uniform-mesh simulation to the errors produced in the variable-mesh simulation. 